\title{
Progress Report on
}

\section{Particle Dynamics in a Wave with Variable Amplitude}

\author{
John R. Cary
}

Principal Investigator

Department of Astrophysical, Planetary, and Atmospheric Sciences

University of Colorado

Boulder, CO 80309-0391

\author{
January, 1992
}

\section{DISCLAIMER}

This report was prepared as an account of work sponsored by an agency of the United States Government. Neither the United States Government nor any agency thereof, nor any of their exployees, makes any warranty, express or implied, or assumes any legal liability or responsiemployect, or bility for the accuracy, completeness, or usefulness of any information, apparatus, product, process disclosed, or represents that its use would not infringe privately owned rade name, trademark, ence herein to any specific commercial product, process, or service by trade nats endorsement, recommanufacturer, or otherwise does not necessarily constitute or imply its endorsement, The views mendation, or favoring by the United States Government or any agency thereof. The views and opinions of authors expressed herein do not United States Government or any agency thereof. 


\begin{abstract}
Our past research efforts led to the derivation of the adiabatic invariant in spatially varying accelerator structures, to the calculation of the loss of the invariant due to trapping, and to a method for determining transverse invariants using a nonperturbative approach to the Hamilton-Jacobi equation. These research efforts resulted in the training of two graduate students who are now working in the area of accelerator physics.
\end{abstract}




\section{Table of Contents}

Abstract ...................................................................

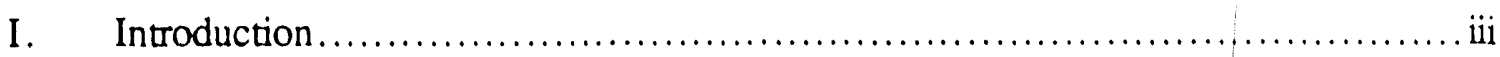

II. Spatial adiabatic trapping and emittance growth due to separatrix crossing: applications to free-electron lasers ............................................

A. Background and Accomplishments.....................................

B. Recent Accomplishments ........................................... 12

III. Diffusive models of tracking results ...................................... 19

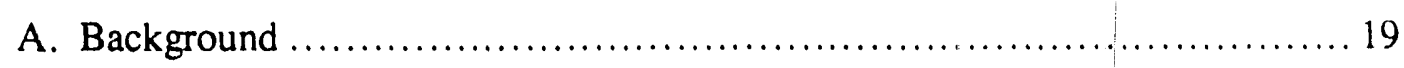

B. Accomplishments. ............................................ 21

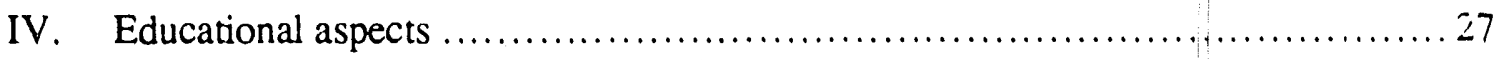

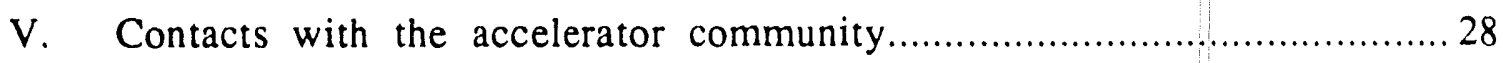

VI. References ........................................................ 29

Appendix A. Particle Dynamics in a Large-Amplitude Wavepacket

Appendix B. Adiabatic Invariance for Spatially Dependent Accelerating \$tructures

Appendix C. Report of the Working Group on the Nonlinear Analysis of Beams

Appendix D. The Plot widget 


\section{Introduction}

Our past research efforts led to several advances in the field of the dynamics of particles in accelerators. We derived the adiabatic invariant for accelerating structures with slow spatial variation. ${ }^{1,3}$ This invariant is useful for understanding trapping in, e.g., radiofrequency quadrupoles. We further analyzed the loss of the adiabatic invariant due to the trapping process (separatrix crossing) in spatially varying systems. ${ }^{1,4}$ We also developed nonperturbative Hamilton-Jacobi methods for determining transverse invariants in nonlinear transport systems. ${ }^{5}$ These research efforts resulted in the training of two graduate students, David Bruhwiler and Bill Gabella, who are now working in the area of accelerator physics.

The research program is broad. Some of our efforts, such as that to determine whether tracking data for particle loss can be modeled by a diffusion equation, are direct applications to accelerators. Other efforts, such as the investigation of symplectic integrators, are more fundamental, but still relevant to accelerator physics. Discussing all of our projects would make this report unwieldy. So instead we concentrate on two of our projects: development of a free-electron laser experiment to test adiabatic trapping theory and modeling SSC tracking data by a diffusive model.

Our recent work on adiabatic theory has been to design a free-electron laser experiment to test adiabatic trapping theory. Separatrix crossing theory predicts that the emittance growth due to trapping in a free-electron laser can be greatly diminished by trapping adiabatically. This would be especially advantageous in a recirculating freeelectron laser, where the longitudinal emittance growth due to each interaction of the beam with the wiggler must be eliminated by cooling. ${ }^{6}$ Less cooling would be needed if adiabatic trapping were used. 
A possible location for such an experiment would be at the Accelerator Test Facility at Brookhaven National Laboratory, where there is a high-brightness electron beam of energy up to $50 \mathrm{MeV}$. As there is also a $\mathrm{CO}_{2}$ laser at this facility, only an appropriate wiggler would be needed. Preliminary considerations show that adiabatic trapping and detrapping wiggler can be made that would be of the order of 2 meters long.

Our research to model tracking data by a diffusive model has the goal of understanding whether simple chaotic diffusion underlies the results of tracking calculations. In the tracking calculations, particles are launched at various distances from the design orbit. On average the confinement time increases as the launch point of the particles is moved towards that of the design orbit. However, the confinement time is not unique, because trajectories launched at the same distance, but at different locations around the ring, have different confinement times. The presence of this statistical aspect leads one to believe that the loss may be described by a chaotic diffusion, as is the case in other dynamical systems. We have been developing a method for determining whether chaotic diffusion is consistent with SSC tracking data. We show that there is a consistency relation among the first three moments of the con'inement time if the loss is describable by a diffusion equation. We are in the midst of developing computer codes to see whether this consistency relation is satisfied for the given tracking data.

The ultimate goal of this research is to seek a dynamical basis for the diffusior. equation, as has been found in other cases. ${ }^{7}$ Such a dynamical basis would allow one to derive the diffusion coefficient. Use of the diffusion equation rather than tracking data would greatly decrease the computational requir ments for accelerator simulation. Moreover, with an understanding of the basic process, methods for diminishing the chaotic loss might be found.

To maintain strong ties between our university based accelerator research program 
and the accelerator research at the national laboratories and other universities, we have continued to maintain a high level of contact with the accelerator community. An example is this year's sabbatical of the principal investigator at Fermilab. This sabbatical enabled the P.I. to interact with the accelerator physicists of the Accelerator Physics group led by Steve Peggs. In conjunction with Leo Michelotti and Jim Holt, the P.I. developed software for mouse-click interaction with tracking codes. With Bill Gabella, this was used to study noise extraction of the beam periphery for a possible parasitic fixed-target experiment at the SSC. Documentation of this software is included in an appendix.

This research program has also been instrumental in training new accelerator physicists. As mentioned above, past funding allowed two graduate students to receive their Ph.D.'s in accelerator physics. Two new graduate students began this last year. Both are likely to finish their theses in 2-4 years. The current funding also provides for one postdoctoral fellow. This person, Carson Chow, completed his thesis in plasma physics and nonlinear dynamics. During his fellowship at the University of Colorado, Carson Chow will be able to learn the techniques and jargon of accelerator physics while doing research in this area. All three of these researchers will be attending the school on accelerator physics this summer.

In the following three sections we provide brief overviews of two areas of the proposed research: experimental tests of adiabatic trapping theory and diffusion models for SSC tracking data. Section IV discusses the educational aspects of this research program, while Sec. $V$ notes the contacts made between the present research group and the accelerator community at large. The references are contained in Sec. VI. The appendices contain reports produced under past years of this research program. 


\section{Spatial adiabatic trapping and emittance growth due to separatrix crossing: applications to free-electron lasers}

In previous years of this research project we developed the theory of spatial adiabr, acity. We calculated the adiabatic invariant applying to the situation when an acce'.rating structure varies slowly in space rather than time. We also showed how to calculate the increase of emittance due to separatrix crossing. We have begun to design experiments to test these analyses. Our preliminary calculations show that these tests could be carried out in a free-electron laser experiment at the Accelerator Test Facility at Brookhaven National Laboratory. ${ }^{8}$ If successful, such experiments would point to a way of ciesigning a recirculating free-electron laser with diminished beam cooling requirements, as sufficiently adiabatic trapping gives arbitrarily small emittance increase.

\section{A. Background and Accomplishments}

For a Hamiltonian $\mathrm{H}(\mathrm{q}, \mathrm{p}, \varepsilon t)$ depending slowly on the time variable, the action,

$$
\left.\mathrm{I}(\mathrm{E}, \mathrm{\varepsilon t}) \equiv \oint \mathrm{p}\right|_{\mathrm{H}=\mathrm{E}} \mathrm{dq},
$$

the phase-space area enclosed by a closed contour of $\mathrm{H}$ at fixed time, is the adiabatic invariant. Slowly means that the particle executes many oscillations before the parameters of the Hamiltonian change significantly. Invariance of the action, $I\left(E_{i}, \varepsilon t_{i}\right)=I\left(E_{f}, \varepsilon t_{f}\right)$, allows one to solve for the energy at one time in terms of the energy at another time. Adiabatic theory has also been used to predict the phase-space area occupied by a beam adiabatically trapped in an accelerating potential.

\section{Spatial adiabatic invariance}

We have analyzed the case of adiabatic, spatially varying, accelerating structures. 
We have shown that the usual adiabatic invariants for the temporally varying case are not invariant in the spatially varying case. This affects, in particular, the arguments that give the area occupied by a beam that has been adiabatically trapped in a spatially varying structure. Our results apply to a large number of accelerator and plasma physics problems such as trapping in the accelerating potential of an $\mathrm{RFQ},{ }^{9}$ in the ponderomotive (decelerating) potential of free-electron lasers, ${ }^{10}$ in plasma heating ${ }^{11}$ and in plasma turbulence. $^{12}$

For such structures, the potential instead has an amplitude that varies spatially:

$$
H(q, p, t)=\frac{p^{2}}{2 m}+e \Phi(\varepsilon q) \cos (k(q-u t))
$$

Now the particles see an amplitude growing as they enter the accelerating structure. After being trapped in a stable bucket they may be accelerated or decelerated depending on how the phase velocity $u$ changes with position. A model potential is shown in Fig. 2.1.

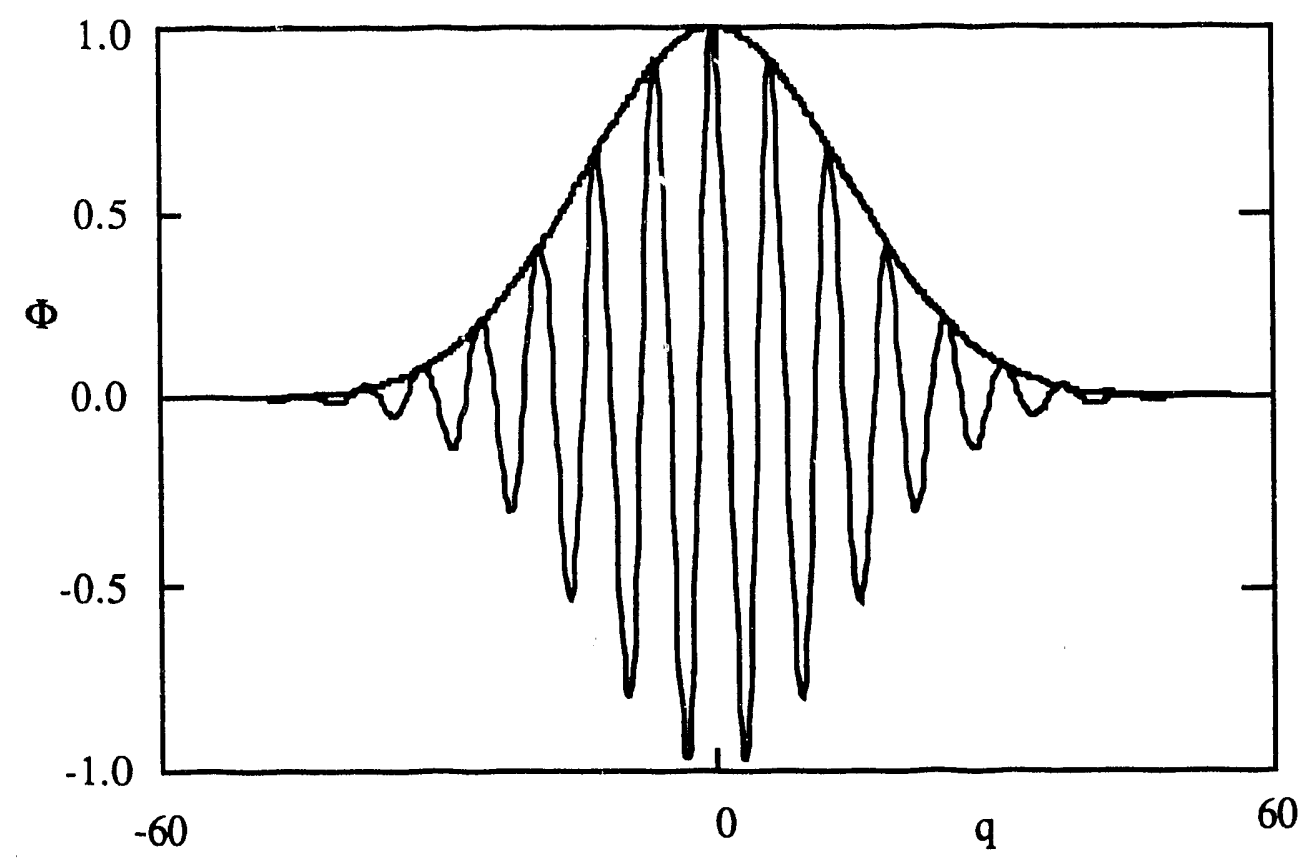

Fig. 2.1. Potential for (2.2) versus coordinate. 
Temporal adiabatic theory does not apply to this situation. One can see this by considering the case $u=0$, where the Hamiltonian is time independent and so $H$ is conserved. Temporal adiabatic theory would say that for passing particles, the actions $\Psi_{ \pm}(E, \varepsilon q)$, obtained by integrating along a contour of $\mathrm{H}$ either above $(+)$ or below $(-)$ the bucket separatrix at $\mathrm{H}=\mathrm{e} \Phi$, are invariants. However, if both $\Psi_{ \pm}$and $\mathrm{E}$ are constant, then $\mathrm{q}$ must be constant if $\mathrm{L}_{ \pm}(\mathrm{E}, \varepsilon q)$ has a nontrivial dependence on $\mathrm{q}$. This contradiction shows the previous adiabatic theory to be false.

In previous work ${ }^{2,3}$ we showed that one could find the new adiabatic invariant by transforming to the new fast variable $\mathrm{q}-\mathrm{ut}$, which has the conjugate momentum $\mathrm{z} \equiv \mathrm{p}+\mathrm{K} / \mathrm{u}$, where

$$
\mathrm{K}(\mathrm{q}-\mathrm{ut}, \mathrm{p}, \varepsilon \mathrm{q}) \equiv \frac{(\mathrm{p}-\mathrm{mu})^{2}}{2 \mathrm{~m}}+\mathrm{e} \Phi(\varepsilon \mathrm{q}) \cos (\mathrm{k}(\mathrm{q}-\mathrm{ut}))
$$

is the new Hamiltonian for these variables, and $\varepsilon q$ is the new slowly varying time. One can see that $\mathrm{K}$ is the Hamiltonian in the frame moving with the phase of the wave. The adiabatic invariant is then given by the integral,

$$
J=\int(p+K / u) d(q-u t)=\int p d(q-u t)+(K / u) \int d(q-u t)
$$

along a contour of constant $\mathrm{K}$ with the slow spatial dependence $\varepsilon q$ held fixed.

For trapped particles, the phase (q-ut) begins and ends at the same point. Hence, the second term vanishes and we have,

$$
\mathrm{J}_{\mathrm{T}}=\oint \mathrm{pd}(\mathrm{q}-\mathrm{ut}) \equiv 2 \mathrm{I}(\varepsilon \mathrm{q}, \mathrm{K})
$$

The last equality defines the variable I, which for trapped particles is simply half the enclosed area. (This factor of $1 / 2$ makes I a continuous phase-space variable.) Thus, the 
trapped-particle spatial adiabatic invariant is also the trapped-particle temporal adiabatic invariant. This must be, since a trapped particle trajectory, being confined to a single bucket, does not sample enough of the potential to determine whether the amplitude is spatially or temporally varying. Because $I$ is half the temporal adiabatic invariant for trapped particles, and it is the temporal adiabatic invariant for passing particles, we will call I the action.

For plassing particles, the phase q-ut does not begin and end at the same value, but changes by $2 \pi / \mathrm{k}$. Thus, the adiabatic invariant for \pm passing particles is given by

$$
\mathrm{J}_{ \pm}=\mathrm{I}+2 \pi \mathrm{H} / \mathrm{ku}
$$

with

$$
I(\varepsilon q, H) \equiv \int_{0}^{2 \pi}(p-u) d(q-u t)
$$

being the integral along a contour of constant $\mathrm{K}$. The convention chosen here of integrating in the direction of increasing phase q-ut makes I a signed quantity; it is positive (negative) for positive (negative) passing particles.

\section{Adiabatic invariant change due to separatrix crossing}

In the early years of this line of research, we pioneered the theory of adiabaticity loss tue to separatrix crossing in adiabatic systems. We review this theory first for the case of a Hamiltonian, $\mathrm{H}(\mathrm{q}, \mathrm{p}, \mathrm{\varepsilon t})$, with one degree of freedom and slow time-dependence, i.e. small $\varepsilon$. Adiabatic invariance theory states as the contours of $\mathrm{H}$ change in the course of time, for small $\varepsilon$ a given trajectory can always be found on a contour containing the same area as did the contour of the trajectory at the initial time. The requirement for $\varepsilon$ is that it must be small compared with the orbit frequency $v_{0}$ calculated $b: \cdot$ neglecting the change of the Hamiltonian function with time. 
Unfortunately, adiabatic invariance breaks down for particles near the separatrix. (A separatrix is a contour of $\mathrm{H}$ containing an $\mathrm{x}$-point, a saddle point, which is a fixed point of the dynamics for $\varepsilon=0$. Contours of a typical Hamiltonian with a separatrix are shown in Fig. 2.2.) This causes adiabatic invariance to break down in a large number of cases, because a separatrix can sweep across large regions of phase space in the course of time. For example, if the separatrix in Fig. 2.2 is growing in time, it collides with a trajectory in region-c at the time $t_{x}$ given by $Y_{c}\left(\varepsilon t_{x}\right)=J_{i}$, in which $Y_{c}\left(\varepsilon t_{x}\right)$ is the area enclosed by the entire separatrix, and $J_{i}$ is the initial value of the adiabatic invariant. This time $t_{x}$ is called the pseudo-crossing time. In the vicinity of this time the adiabatic invariant is inapplicable.

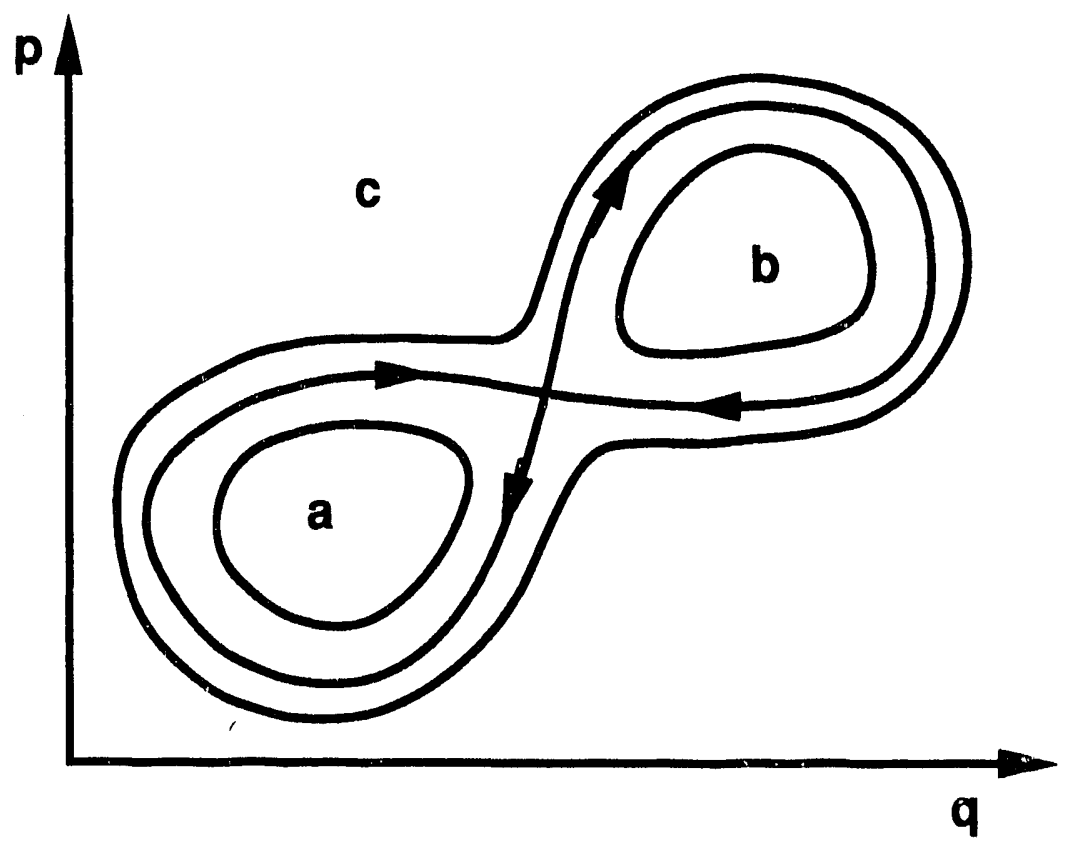

Figure 2.2. Contours of a typical Hamiltonian with a separatrix.

We analyzed the consequences of crossing a separatrix in detail previously. ${ }^{13}$ It was found that the new value of the adiabatic invariant depends on the lobe of the separatrix into which the trajectory becomes trapped. For a trajectory becoming trapped into, say, lobe-a, (See Fig. 2.2) the new value of the adiabatic invariant, $J_{f a}$, is close to $Y_{a}\left(\varepsilon t_{x}\right)$, the phase-space area enclosed by lobe-a of the separatrix at tise pseudo-crossing time. The deviation, $\delta J_{\mathrm{fa}}\left(M_{\mathrm{c}}\right) \equiv \mathrm{J}_{\mathrm{fa}}-\mathrm{Y}_{\mathrm{a}}\left(\varepsilon \mathrm{t}_{\mathrm{x}}\right)$, is found to depend on a crossing parameter $\mathrm{M}_{\mathrm{c}}$, 
which is related to the initial phase, or adiabatic angle, of the trajectory. This implies that an ensemble initially distributed on an adiabatic invariant ring, a single contour, is ultimately distributed throughout an adiabatic invariant annulus after a separatrix crossing. Thus, separatrix crossing leads to a spread of the adiabatic invariant.

In previous years of this research, ${ }^{4}$ separatrix crossing theory was used to calculate the increase of emittance of particles repeatedly trapping and detrapping in a wave. Separatrix crossing theory provides an analytic expression for the increase of emittance at each crossing. Summing the increases with the random-phase approximation gave an effective diffusion coefficient describing the long-time rate of emittance growth.

More recently, separatrix crossing theory was applied to problems with spatial adiabaticity. ${ }^{1}$ We explicitly calculate the growth of emittance for a set of trajectories passing through accelerating fields of the form (2.2). We show, by comparison with direct numerical integration, that the analytic theory is accurate in the appropriate regime, i.e., when the typical synchrotron length is small comparea with the scale length of the wavepacket.

\section{B. Recent Accomplishments}

\section{Predictions of spatial adiabatic theory for longitudinal acceptance}

In the past two years we investigated in detail the consequences of spatial adiabatic theory for trapping in spatially varying structures. We found the a beam of particles of incoming momentum does not trap at the same amplitude as is found from temporal adiabatic theory. Instead, both the location and the trapping capability of a wave of some given amplitude are affected. In particular, there exist values of incoming momentum which cannot be trapped. These results are discussed in some detail in Refs. 12 and 13. Below we summarize these results. 
We consider a beam of particles with momentum $p_{i}=m(u+\Delta)$ far from the accelerating structure. Such particles are in the positive passing state and have initial value of the adiabatic invariant,

$$
\mathrm{J}_{+\mathrm{i}}=(2 \pi \mathrm{m} / \mathrm{k})(\Delta+\mathrm{K} / \mathrm{mu})=(2 \pi \mathrm{m} / \mathrm{k})\left(\Delta+\frac{1}{2} \Delta^{2} / \mathrm{u}\right)
$$

The last equality fnllows from the fact that $K=(\mathrm{p} \mathrm{mu})^{2} / 2 \mathrm{~m}$ far from the accelerating structure. These particles trap when the value,

$$
Y_{+}=8 \mathrm{~mA} \mathrm{~A}^{1 / 2} / \mathrm{k}+2 \pi \mathrm{mA} / \mathrm{ku}
$$

of the positive passing adiabatic invariant on the separatrix equals the initial value of the adiabatic invariant. Equating (2.8) and (2.9) shows that these particles become trapped at amplitude $A_{x}$ satisfying

$$
\Delta+\frac{1}{2} \Delta^{2} / \mathrm{u}=4 \mathrm{~A}_{\mathrm{x}}^{1 / 2} / \pi+\mathrm{A}_{\mathrm{x}} / \mathrm{u}
$$

which implies that these particles are spread throughout an area of

$$
a_{q+}(\Delta)=16 m A_{x}^{1 / 2} / k=(32 m u / k \pi)\left(\sqrt{1+\pi^{2} \Delta / 4 u+(\pi \Delta / 2 u)^{2} / 2}-1\right),
$$

which is the area enclosed by the accelerating bucket at the position of trapping. For small values of the momentum difference $\Delta$ (and, therefore, trapping amplitude), the result is identical to that obtained in the temporally varying case. However, for large positive values of $\Delta / u$ one finds a correction of the order of $10 \%$.

For particles with negative values of $\Delta$, the difference between the spatially and temporally varying cases is much greater. Such particles trap when the initial value, $\mathrm{J}_{-\mathrm{i}}$, of the negative-passing-state adiabatic invariant, which is also given by $(2.8)$ far from the 
accelerating structure, matches the value,

$$
Y_{-}=-8 m A^{1 / 2} / k+2 \pi m A / k u
$$

of the negative-passing-state adiabatic invariant on the separatrix. Equating (2.8) and (2.12) gives

$$
\mathrm{a}_{\mathrm{q}-}(\Delta)=16 \mathrm{~mA}_{\mathrm{x}}^{1 / 2} / \mathrm{k}=(32 \mathrm{mu} / \mathrm{k} \pi)\left(1 \pm \sqrt{1+\pi^{2} \Delta / 4 \mathrm{u}+(\pi \Delta / 2 \mathrm{u})^{2} / 2}\right) .
$$

The sign of the radical must be chosen such that the area is positive.

For small, negative $\Delta$, the condition of positive area indicates that the minus sign must be chosen in (13). This sign continues to be valid until the radicand becomes negative, just beyond which there is no solution. The range in which there is no solution is

$$
|\mathrm{p}|<\left(1-8 / \pi^{2}\right) \mathrm{mu}
$$

Thus, initial momenta in this range can never be trapped. They are always reflected by the ponderomotive potential before colliding with the separatrix. This effect never occurs in the temporally varying case.

For still more negative values of the momentum, $p<\left(8 / \pi^{2}-1\right) \mathrm{mu}$, particles entering the accelerating structure from the left will interact with the separatrix if the potential amplitude is large enough, but only in becoming untrapped there, as a more detailed aralysis $^{11}$ shows. The untrapped particles originate from positive-passing incident particles that pass through the separatrix to become reflected, negative-passing particles. We call this resonant reflection.

We have seen that spatially adiabatic systems can be quite different from temporally adiabatic systems. In temporally adiabatic systems all particles can be trapped if the 
amplitude is increased sufficiently. In contrast, no particles in a spatially adiabatic system with momentum less than $\left(1-8 / \pi^{2}\right)$ mu can be trapped by the potential, no matter how large it becomes. Moreover, in spatially adiabatic systems, there is the new phenomenon of resonant reflection.

\section{Estimates of experimental parameters needed to observe odiabatic trapping}

We would now like to see if experimental tests of adiabatic trapping theory can be performed. Adiabatic theory predicts that that the increase of longitudinal emittance of a beam in trapping process is proportional to the adiabaticity parameter. The adiabaticity parameter is proportional to the characteristic crbit period divided by the characteristic time over which the trapping takes place. For example, for trapping in a longitudinal bucket with synchrotron bounce period $\lambda_{b}$ over a length $\mathrm{L}$, the emittance increase is proportional to $\lambda_{\mathrm{b}} / \mathrm{L}$.

Experimental tests are needed to see whether such theory is relevant to a real situation, in which many real-world effects, such as transverse motion and experimental errors and unknowns, come irito play. As the Accelerator Test Facility at Brookhaven National Laboratory is nearing completion, and it provides many of facilities needed for such tests, we decided to investigate whether tests of adiabatic theory could be carried out there.

The Accelerator Test Facility (ATF) has both a high-power $\mathrm{CO}_{2}$ laser and an electron linac capable of producing electrons with energies of five to $50 \mathrm{MeV}$. Combining these two items with an appropriate wiggler produces a free-electron laser amplifier with a large ponderomotive potential. The adiabaticity requirement is that the synchrotron length be short compared with the scale length of the ponderomotive potential. Thus, our calculations have been geared towards finding an experimental setup within the restrictions of available laser power and beam energy such that an adiabatic wiggler could be 
constructed.

In a free-electron laser, an electron beam of relativistic factor $\gamma$ passes through a spatially oscillating magnetic field of wavelength $\lambda_{\mathrm{w}}$. The strength of the magnetic field is represented by the dimensionless vector potential,

$$
a_{w}=\frac{e B_{w}}{m k_{w} c^{2}}
$$

where $k_{w} \equiv 2 \pi / \lambda_{w}$ and $B_{w}$ is the peak wiggler field. If a laser of wavelength $\lambda_{s}$ is present, a ponderomotive potential is produced. This potential is resonant with the electrons when ${ }^{14}$ the condition,

$$
\lambda_{\mathrm{s}}=\lambda_{\mathrm{w}} / 2 \gamma_{\mathrm{r}}^{2}
$$

is satisfied, where the reduced relativistic factor is given by

$$
\gamma_{\mathrm{r}}=\gamma /\left(1+\frac{1}{2} \mathrm{a}_{w}^{2}\right)^{1 / 2}
$$

for a linearly polarized wiggler. If the electrons are trapped, the wavelength $\lambda_{\mathrm{w}}$ can be decreased, while adiabaticity guarantees that Eq. (2.16) remains satisfied. Thus, the electrons lose energy, which is transferred to the laser light.

The adiabaticity requirement is satisfied if the electrons execute many synchrotronbounce oscillations in the time for the beam to slow down. The synchrotron bounce wavenumber is given by

$$
k_{b}^{2}=\frac{e^{2} B_{w} E_{s}}{m^{2} c^{4} \gamma_{r}^{2}},
$$

where $E_{S}$ is the peak laser electric field in cgs units. Thus, adiabaticity is satisfied when 
$\lambda_{\mathrm{b}} \ll<\mathrm{L}$, where $\mathrm{L}$ is the characteristic length over which the system chlinges, and $\lambda_{b} \equiv 2 \pi / k_{b}$ is the synchrotron wavelength.

This same condition is required for adiabatic trapping. To satisfy this colfdition, we imagine a constant laser power along the electron beam. The ponderomotive potential, proportional to the product of the laser amplitude and the wiggler amplitude, is constructed to increase slowly over a length $L$ by having the wiggler amplitude vary slowly. This implies that we want $\lambda_{b}$ to be as small as possible. Thus, we need large laser power and wiggler strength, but low $\gamma$.

A preliminary design was arrived by the following procedure. The $\mathrm{CO}_{2}$ laser wavelength is $10.6 \mu \mathrm{m}$. To keep the relativistic factor low, we chose $\lambda_{w} \# 0.8 \mathrm{~cm}$, consistent with the wiggler built by Stoner. ${ }^{15}$ Equation (2.16) then implies $\gamma_{\mathrm{T}}{ }$ 19.8, or that the electron beam have energy $9.6 \mathrm{MeV}$. We set the wiggler strength to be $5 \mathrm{k} / \mathrm{j}$, again consistent with Stoner's design. Next we decided on a synchrotron wavelength of $22 \mathrm{~cm}$. This would allow 9 synchrotron wavelengths across a 2 meter wiggler. Equation (2.18) then implies that the laser field must be $1.8 \times 10^{4}$ statvolt $/ \mathrm{cm}$.

The laser field is limited by the laser power and the laser spot size. We chose the laser power to by $10 \mathrm{GW}$ as is expected at ATF. This was used in the relation $P_{S}=c\left(a_{s}\right)^{2} / 8$ between the power, the laser spot size a, and field strength to determinite the spot size. We obtained a spot size of $2 \mathrm{~mm}$. This implies a Rayleigh length of $78 \mathrm{~cm}$, which, since the ATF laser is diffraction limited, implies that the iaser beam will not spread excessively from its minimum spot size over the length of the wiggler.

We also checked whether such a configuration would have a sufficiently large bucket for trapping the electrons. The ponderomotive potential amplitude $\mathrm{V}$ is related to the synchrotron bounce wavenumber by 


$$
\mathrm{k}_{\mathrm{b}}=\left(\mathrm{k}_{\mathrm{s}}^{2} \mathrm{~V} / \mathrm{m} \gamma_{\mathrm{r}}^{3}\right)^{1 / 2} / \mathrm{c}
$$

This is the standard result for a particle of mass $m \gamma_{r}^{3}$ moving at velocity $c$ bouncing in a longitudinal potential of amplitude $\mathrm{V}$ and wavenumber $\mathrm{k}_{\mathrm{s}}$. The bucket height in momentum in this situation is given by $\Delta \mathrm{p}^{2} / 2 \mathrm{~m} \gamma_{\mathrm{r}}^{3}=\mathrm{V}$. From this one can show that the relative bucket height is given by

$$
\Delta \mathrm{p} / \mathrm{p}=2 \gamma_{\mathrm{r}}^{2} \lambda_{\mathrm{s}} / \lambda_{\mathrm{b}}
$$

For the situation described above, the relative bucket height is $4 \%$. This is much greater than the expected $0.3 \%$ energy spread of the ATF beam.

Lastly, we checked whether the beam's finite radius, which cause different beam particles to see different wiggler strengths, would cause significant variation of the reduced relativistic factor. For the case just discussed, the dimensionless vector potential is 0.37 . Hence, even at $100 \%$ variation of the wiggler strength, the divisor in Eq. (2.17) varies by only $2 \%$. This effect is therefore expected to be negligible. 


\section{Diffusive models of tracking results}

The purpose for the following work is to discover whether particle loss in an accelerator can be described.by existing models of chaotic diffusion. We plan to answer this question by testing the consistency of such models against tracking code results. The success of a diffusive model will give the properties of particle loss in terms of a diffusion coefficient. Therefore, a diffusive model will greatly reduce the effort and time needed to evaluate the quality of beam transport lines.

\section{A. Background}

At present tracking codes are used to determine the quality of beam Iransport lines. Tracking codes follow the particles element by element around the accelerator. In Fig. 3.1 is a sketch of tracking results for the SSC. ${ }^{16}$ The abscissa is the launch betatron amplitude. The ordinate is the confinement time of an orbit, measured in circuits around the machine. This figure shows that the confinement time of particles increases exponentially with launch betatron amplitude, A. This figure also shows that there are several values of the confinement time for each launch amplitude. This happens because particles may be launched at the same amplitude, but with different phases, i.e., at different locations along the machine.

This process is statistical in the same way that many chaotic systems are statistical: Nearby orbits can evolve very differently. So we now ask whether these results can be described by a diffusion equation, ${ }^{4,7}$ in parallel with previous work in nonlinear dynamics. In this line of research, there is an underlying approximate invariant, J. Perturbations cause the motion to look like diffusion in this approximate invariant, so that the distribution function obeys the equation, 


$$
\frac{\partial \mathrm{f}}{\partial \mathrm{t}}=\frac{\partial}{\partial \mathrm{J}}\left(\mathrm{D}(\mathrm{J}) \frac{\partial \mathrm{f}}{\partial \mathrm{J}}\right)
$$

In fact, this special form of the diffusion equation applies only when the variable $J$ is one in which the steady-state solution for particles trapped in some region is of constant density. Thus, for an ergodic Hamiltonian system, from which one obtains (3.1) by integrating out other degrees of freedom, the differential element $\mathrm{d} J$ must be proportional to the volume of phase space in the differential rigion between surfaces $\mathrm{J}$ and $\mathrm{J}+\mathrm{dJ}$.

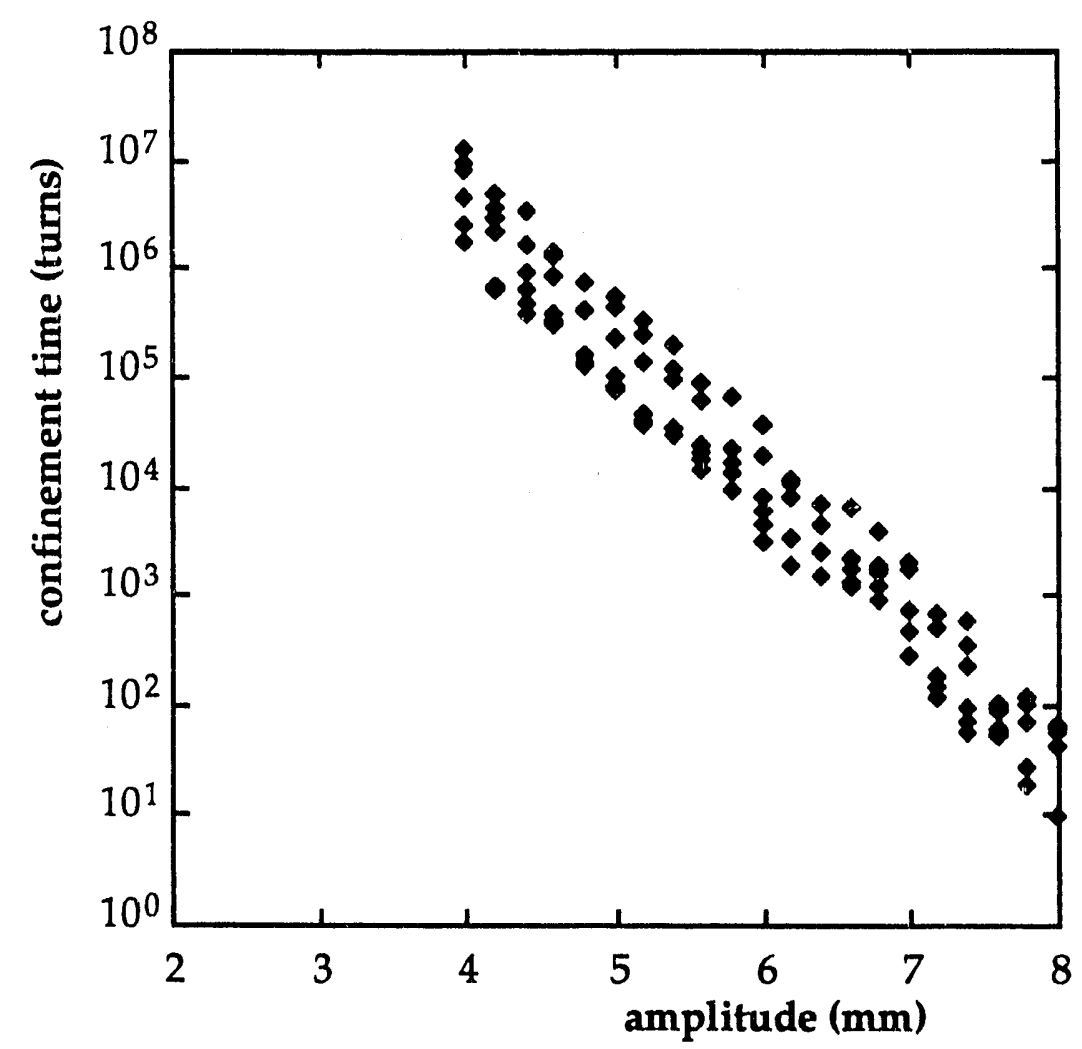

Fig. 3.1. Sketch of confinement results from the tracking codes of the SSC group.

Two functions must be known to have a diffusion equation (3.1) for these tracking results. The first is the diffusion coefficient $D(J)$. In other dynamical problems ${ }^{7}$ this diffusion coefficient has been determined from first principles. Here the goal is to determine the coefficient from the data. The second unknown function $J(A)$ relates the phase-space variable $J$ to the betatron launch amplitude $A$. Were the underlying approximate invariant the linear betatron invariant for two coupled transverse degrees of 
freedom, the phase space volume, that of a four dimensional ellipsoid, would be proportional to $\mathrm{A}^{4}$. However, here we hope to determine $\mathrm{J}(\mathrm{A})$ also from the data.

\section{B. Accomplishments}

Our goal is to determine whether the tracking data of Fig. 3.1 are consistent with the diffusion model (3.1). To do so, we must first determine the two arbitrary functions, $\mathrm{D}(\mathrm{J})$ and $\mathrm{J}(\mathrm{A})$. Then we must use these functions to make a prediction about the data. If the prediction is consistent, we would then consider trying to find such a diffusion model from first principles.

Our method will be to consider the confinement-time moments as a function of the launch amplitude. The diffusion model makes a prediction for all such moments. By using two such moments, we will be able to deduce the two functions, $D(J)$ and $J(A)$. Comparison of the prediction of the third moment with the result from tracking data will determine consistency. Hence, our first goal is to show how these moments rely on the chosen diffusion equation.

To solve the diffusion equation, we must decide on boundary conditions. Figure 3.1 shows that for smaller $\mathrm{A}$ the confinement time is very large. This implies that the diffusion coefficient is becoming very small. For the trajectories launched at larger amplitudes the precise small-amplitude dynamics are unimportant, so we can just as well assume that there is a "hard" wall at some minimum value $A_{\min }$ of the amplitude. This corresponds to choosing the boundary condition,

$$
\left(\mathrm{D}(\mathrm{J}) \frac{\partial \mathrm{f}}{\partial \mathrm{J}}\right)_{\mathrm{J}_{\min }}=0
$$

At large amplitudes the trajectories are lost in just a few turns. Hence, we can assume there is a loss boundary at $\mathrm{J}_{\max }$ at which the boundary condition, 


$$
\mathrm{f}\left(\mathrm{J}_{\max }\right)=0
$$

holds. Here $\mathrm{J}_{\min } \equiv \mathrm{J}\left(\mathrm{A}_{\max }\right)$.

We are now ready to calculate the confinement-time moments. We define the function $\mathrm{F}\left(\mathrm{J}, \mathrm{t} ; \mathrm{J}_{0}\right)$ to be the solution to Eq. (15) satisfying

$$
\mathrm{F}\left(\mathrm{J}, 0 ; \mathrm{J}_{0}\right)=\delta\left(\mathrm{J}-\mathrm{J}_{0}\right)
$$

This is just the Green's function for this system. If we know $F$, then we can calculate an arbitrary evolution and, hence, all of the confinement-time moments. However, instead of calculating $F$, we will derive a recursive relation between successive moments.

The probability for particles launched at $\mathrm{J}_{0}$ to remain in the system at time $t$ is

$$
P\left(t, J_{0}\right) \equiv \int_{J_{\min }}^{J_{\max }} \mathrm{dJ} F\left(J, t ; J_{0}\right)
$$

(This probability decays exponentially fast provided the spectrum of the diffusion operator is discrete.) Thus, the loss rate is given by

$$
v \equiv-\frac{\partial P}{\partial t}=-\left(D(J) \frac{\partial F}{\partial J}\right)_{J_{\max }}
$$

The mean loss time,

$$
\tau\left(\mathrm{J}_{0} ; \mathrm{D}\right)=\int_{0}^{\infty} \mathrm{dt} \mathrm{t} v\left(\mathrm{t} ; \mathrm{J}_{0}\right)=\int_{0}^{\infty} \mathrm{dt} \mathrm{P}\left(\mathrm{t} ; \mathrm{J}_{0}\right),
$$

is simply the time weighted by the loss rate. The last equality here follows from integration 
by parts. On left side of Eq. (3.7) we have indicated that the mean loss time is not only a function of the launch position, but also a functional of the diffusion coefficient.

Because Eq. (3.1) is linear and time independent, we introduce the Laplace transform. We get the relation,

$$
s \mathrm{G}=\frac{\partial}{\partial \mathrm{J}}\left(\mathrm{D}(\mathrm{J}) \frac{\partial \mathrm{G}}{\partial \mathrm{J}}\right)+\delta\left(\mathrm{J}-\mathrm{J}_{0}\right)
$$

for the Laplace transform $G$ of the distribution F. Similarly, we introduce the Laplace trans $s^{\text {frrm, }}$

$$
\mu(s) \equiv \int_{0}^{\infty} d t e^{-s t} v(t)=-\left(D(J) \frac{\partial G}{\partial J}\right)_{J_{\max }},
$$

of the loss rate. Differentiation of Eq. (3.9) with respect to $s$ and setting $s$ to zero gives the negative of the mean loss time,

$$
\left.\frac{\partial \mu}{\partial s}\right|_{s=0}=-\int_{0}^{\infty} d t t v(t)=-\tau\left(J_{0} ; D\right)
$$

Combining (24) and (25) gives the mean loss time as

$$
\tau\left(\mathrm{J}_{0} ; \mathrm{D}\right)=\left(\mathrm{D}(\mathrm{J}) \frac{\partial^{2} \mathrm{G}}{\partial \mathrm{s} \partial \mathrm{J}}\right)_{\mathrm{J}_{\max }, \mathrm{s}=0}
$$

The quantity inside the parentheses of Eq. (3.11) can be found by integration of the appropriately differentiated form of Eq. (3.8). We will not repeat all of the details here. The result is 


$$
\tau\left(J_{0} ; D\right)=\int_{J_{0}}^{J_{\max }} d J \frac{\left(J-J_{\min }\right)}{D(J)}
$$

Differentiating this with respect to $\mathrm{J}_{0}$ gives the local relation,

$$
\frac{\partial \tau}{\partial \mathrm{J}_{0}}=\frac{-\left(\mathrm{J}_{0}-\mathrm{J}_{\min }\right)}{\mathrm{D}\left(\mathrm{J}_{0}\right)}
$$

between the diffusion coefficient and the mean confinement time.

Equation (3.13) is the first relation to use in determining whether a diffusion model is applicable. However, we have yet to derive the relation between the betatron amplitude and the invariant $\mathrm{J}$, and we have yet to determine a consistency condition. For this we calculate the higher moments.

We use $\tau_{n}$ to denote the $n^{\text {th }}$ moment moment of the confinement time. This moment is given by

$$
\tau_{\mathrm{n}}\left(\mathrm{J}_{0}\right) \equiv \int_{0}^{\infty} \mathrm{dt} \mathrm{t}^{\mathrm{n}} v\left(\mathrm{t} ; \mathrm{J}_{0}\right)=\left.\left(-\frac{\partial}{\partial \mathrm{s}}\right)^{\mathrm{n}} \mu(\mathrm{s})\right|_{\mathrm{s}=0} .
$$

A recursive formula for $\tau_{n}$ can be deduced from Eq. (3.8), by differentiating with respect to $\mathrm{s}$, setting $\mathrm{s}$ to zero, and using the moment generating function. The result is

$$
\tau_{\mathrm{n}}\left(\mathrm{J}_{0}, \mathrm{D}\right)=\mathrm{n} \int_{\mathrm{J}_{0}}^{\mathrm{J}_{\max }} \frac{\mathrm{dJ^{ \prime }}}{\mathrm{D}\left(\mathrm{J}^{\prime}\right)} \int_{\mathrm{J}_{\min }}^{\mathrm{J}^{\prime}} \mathrm{dJ^{ \prime \prime }} \tau_{\mathrm{n}-1}\left(\mathrm{~J}_{0}, \mathrm{D}\right)
$$

Here we have derived a relation by which one can predict successive moments of the confinement time. Examining the consistency of these moments will determine whether a 
diffusion model exists.

In the last year, we tested the diffusive model by examining higher moments. The procedure for testing the diffusive model is as follows. One fits the function $\tau_{1}\left(A_{0}\right)$ to the tracking results. From this function Eq. (3.13) can be used to determine the diffusion coefficient, $\mathrm{D}\left(\mathrm{J}_{0}\right)$. Next the relation $\mathrm{J}(\mathrm{A})$ is inverted and substituted in Eq. (3.12) giving the predicted second moment. This is then compared to $\tau_{2}\left(\mathrm{~A}_{0}\right)$ from the tracking results.

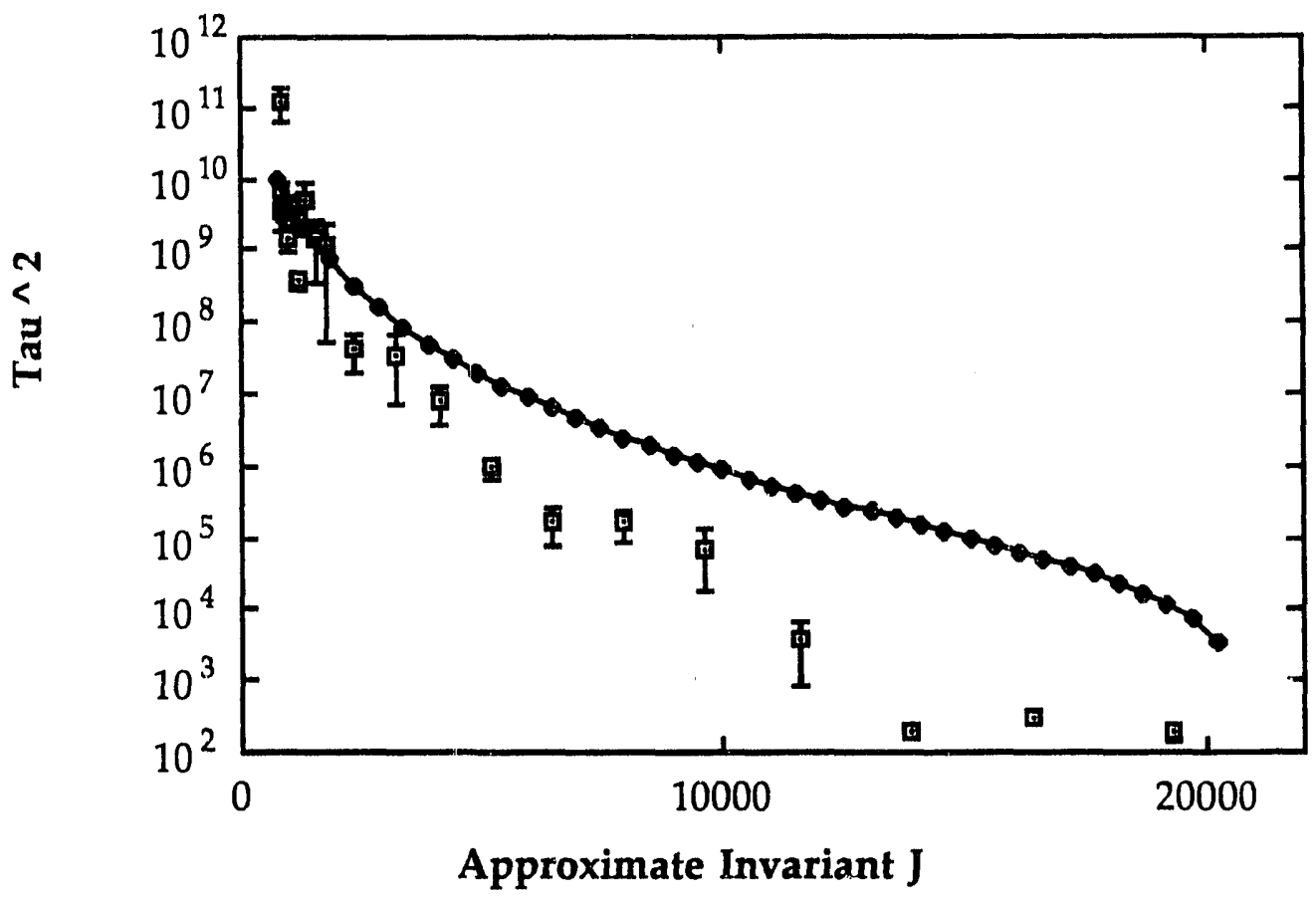

Fig. 3.2 Sketch of second confinement time moments. The solid line shows predicted second mornents for various $\mathrm{J}_{0}$ with $\mathrm{m}=4$. The points are from a tracking code

In performing this test, we assumed a power relation between the variable $\mathrm{J}$ and the launch amplitude $A$,

$$
J=A^{m}
$$

and repeated to test for $m=1,2,3,4$,and 6 . Figure 3.2 shows the results for $m=4$. It can 
be seen from the figure that agreement for $\tau_{2}$ is good for $J_{0}$ near $J_{\min }$, but becomes increasingly poor as $\mathrm{J}_{0}$ approaches $\mathrm{J}_{\max }$. Other values for $\mathrm{m}$ produce similar results. This indicates that if a diffusive rodel exists, a power relation between $\mathrm{J}$ and the launch amplitude $\mathrm{A}$ is not correct.

Therefore we proceed to determine the functional relation

$$
\mathrm{J}=\mathrm{J}(\mathrm{A})
$$

To determine this relationship we start with Eq. (3.12) and $\tau_{2}$ derived from Eq. (3.15). Combining these equations we eliminate the diffusion coefficient and arrive at the following differential equation for $A(J)$,

$$
\frac{\mathrm{dA}}{\mathrm{dJ}}=\frac{2 \tau_{1}-\left(\frac{\mathrm{d} \tau_{2}}{\mathrm{~d} \tau_{1}}\right)_{J_{\max }}+\frac{\mathrm{d} \tau_{2}}{\mathrm{~d} \tau_{1}}}{\left(\mathrm{~J}_{\max }-\mathrm{J}_{\min }\right) \frac{\mathrm{d}}{\mathrm{dA}}\left(\frac{\mathrm{d} \tau_{2}}{\mathrm{~d} \tau_{1}}\right)_{J_{\max }}-\left(\mathrm{J}_{0}-\mathrm{J}_{\min }\right) \frac{\mathrm{d}}{\mathrm{dA}}\left(\frac{\mathrm{d} \tau_{2}}{\mathrm{~d} \tau_{1}}\right)} .
$$

In practice, we fit the first and second confinement time moments to tracking results giving $\tau_{n}(A)$. The derivatives in Eq. (3.18) are them calculated as,

$$
\frac{d \tau_{2}}{d \tau_{1}}=\left(\frac{\frac{d \tau_{2}}{d A}}{\frac{d \tau_{1}}{d A}}\right)
$$

Once Eq. (3.18) is solved, we have the desired relation $A(J)$. Next $D(J)$ is calculated using Eq. (3.13). We then proceed with Eq. (3.15) to test the consistency of the third moment predicted by our theory with that given by the tracking code. 


\section{rV. Educational aspects}

There has always been and will continue to be a strong educational aspect to this research grant. Past funding of this research enabled two students to obtain their Ph. D.'s. The first, David Bruhwiler, has recently taken a job a Grumman in Princeton. He will be working with Ira Lehrman on free-electron lasers. The second, Bill Gabella, is working as a postdoc for Professor Klein of UCLA. Bill is now interacting strongly with the Fermilab Accelerator Physics Department. He is also involved ir calculations for the Super Fixed Target Proposal.

There are now two $\mathrm{Ph}$. $\mathrm{D}$. students associated with this research program. The first, Scott Hendrickson, began working with the PI in January 1991. He has been involved in the calculations on the diffusion description of tracking results and on the freeelectron laser calculations. The second student, Peter Stoltz, began working on accelerator/dyriamics research in June 1991. He has been involved in the calculation of Lyapunov exponents for randomly phased perturbations. He was supported in the summer of 1991 by this grant. He needed no support for this academic year, as he had a two-year fellowship. However, if he is to continue research in accelerator physics, funding for a research assistantship must be provided.

Additionally, this grant provides funding for Carson Chow, the postdoctoral fellow. Dr. Chow comes from MIT, where he completed his thesis in spatio-temporal chaos in plasma turbulence. He, therefore, has a good basic understanding of dynamical phenomena. Comirg to Colorado will provide Dr. Chow with the opportunity to learn accelerator physics. It is planned that his initial project will be to understand nonlinear accelerator transport systems. 


\section{Contacts with the accelerator community}

As the proposed research takes place at a University far from any of the large accelerator laboratories, we have continued to interact strongly through travel and electronic communication. For example, we have obtained SSC tracking data from e-mail from Yiton Yang of SSC. In addition to the regular accelerator conferences, we attended the Accelerator Test Facility review that took place in October of 1991 in order to find out from Drs. Kirk and Ben-Zvi what facilities are available.

At the writing of this report, the PI is on sabbatical at Fermilab, where he is working in the Accelerator Physics Department of the Accelerator Division. During this sabbatical the PI has had the opportunity to interact with many of the accelerator physicists and to learn about accelerator physics operations. The PI did a week in the control room. He became involved in discussions with Pat Colestock, Kathy Harkay, and Andre Gerasimov on collective instabilities in the booster and the Tevatron. He also discussed basic accelerator physics experiments with Todd Satogata and Bill Gabella, an accelerator physicist whose thesis research was supported under this grant.

By far the largest amount of effort was expended in learning about new workstation tools for computational analysis. The PI wrote an interface based on the $\mathrm{X}$ window system for tracking codes. This interface allows a researcher to specify an initial point in phase space by a mouse click in a phase space plot. Simple tracking codes have been grafted onto this interface to study the effects of power supply ripple and external noise on particle confinement. Perhaps unfortunately, because it detracted from physics research, the PI also became involved in installing the appropriate software (Release 4 of version 11 of $\mathrm{X}$ and the Motif widgets and window manager) on the workstations used by the Accelerator Physics Department. This code is included as App. D. 


\section{References}

1 D. L. Bruhwiler, Scattering and Diffusion of Particles in Slowly-Varying LargeAmplitude Waves, Ph. D. thesis, University of Colorado, Boulder (1990).

2 D. L. Bruhwiler and J. R. Cary, Phys. Rev. Lett. (1991), in press. Included as App. A.

3 J. R. Cary and D. L. Bruhwiler, AIP proceedings of 89 US-Japan workshop. Included as App. C.

4 D. L. Bruhwiler and J. R. Cary, Physica 40D, 265 (1989).

5 W. E. Gabella, Numerical Solution of the Hamilton-Jacobi Equation in 2 1/2 Degrees of Freedom, Ph. D. thesis, University of Colorado, Boulder (1991).

6 A. Renieri, Nuovo Cimento 53B, 160 (1979).

7 A. B. Rechester and R. B. White, Phys. Rev. Lett. 44, 1586 (1980); A. B. Rechester, M. N. Rosenbluth and R. B. White, Phys. Rev. A 23, 2664 (1981); J. R. Cary, J. D. Meiss and A. Bhattacharjee, Phys. Rev. A 23, 2744 (1981).

8 I. Ben-Zvi, Bull. Am. Phys. Soc. 36, 1512 (1991).

9 I. M. Kapchinskii and V. A. Teplyakov, Prib. Tek. Eksp. 119, 19 (1970); S. O. Schriber, Proc. 1985 Particle Accelerator Conference (Vancouver, British Columbia, 1985) and references therein.

10 For example, C. W. Roberson and P. Sprangle, Phys. Fluids 1, 3 (1989); N. M. Kroll, P. L. Morton, and M. N. Rosenbluth, IEEE J. Quant. Elec. 17, 1436 (1981).

11 W. M. Nevins, T. D. Rognlien, and B. I. Cohen, Phys. Rev. Lett. 59, 60 (1980).

12 V. Fuchs, V. Krapchev, A. Ram, and A. Bers, Physica D14, 141 (1985).

13 J. R. Cary, D. F. Escande, and J. L. Tennyson, Phys. Rev. A34, 4256 (1986); J. R. Cary and R. T. Skodje, Phys. Rev. Lett. 61, 1795 (1988); J. R. Cary and R. T. Skodje, Physica 36D, 287 (1989). 
14 N. M. Kroll, P. L. Morton and M. N. Rosenbluth, IEEE J. Quantum Electron. 17, (1981); T. J. Orzechowski, B. R. Anderson, J. C. Clark, W. M. Fawley, A. C. Paul, D. Proznitz, E. T. Scharlemann, S. M. Yarema, D. B. Hopkins, A. M. Sessler and J. S. Wurtele, Phys. Rev. Lett. 57, 2172 (1986).

15 R. Stoner, S. C. Chen and G. Bekefi, Bull. Am. Phys. Soc. 36, 2437 (1991).

16 A. Chao, in Nonlinear Dynamics and Particle Acceleration (AIP Conf. Proc. no. 230), (American Institute of Physics, New York, 1991) p. 203. 


\section{Appendix D. The plot widget}

The following is the coding developed for interactive tracking of one and a half degrees of freedom. This code uses the libraries of release 4 of version 11 of the $\mathrm{X}$ window system. Clicking the mouse at a point on the graph initiates a trajectory. The iterates of the trajectory are displayed in the window. 


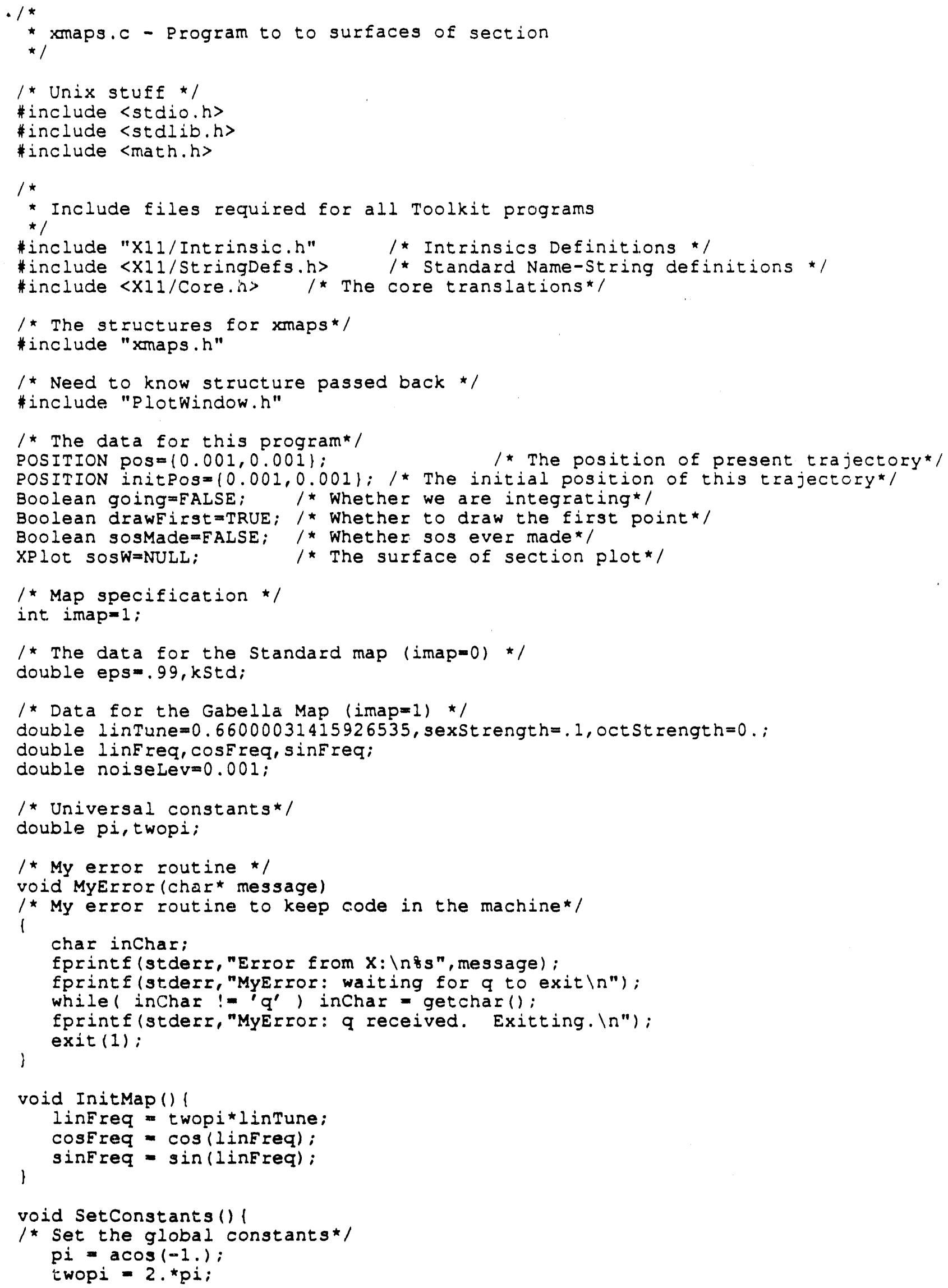







- DrawPoints (sosw, 1, \&posPtr->x, \&posPtr->y);

1

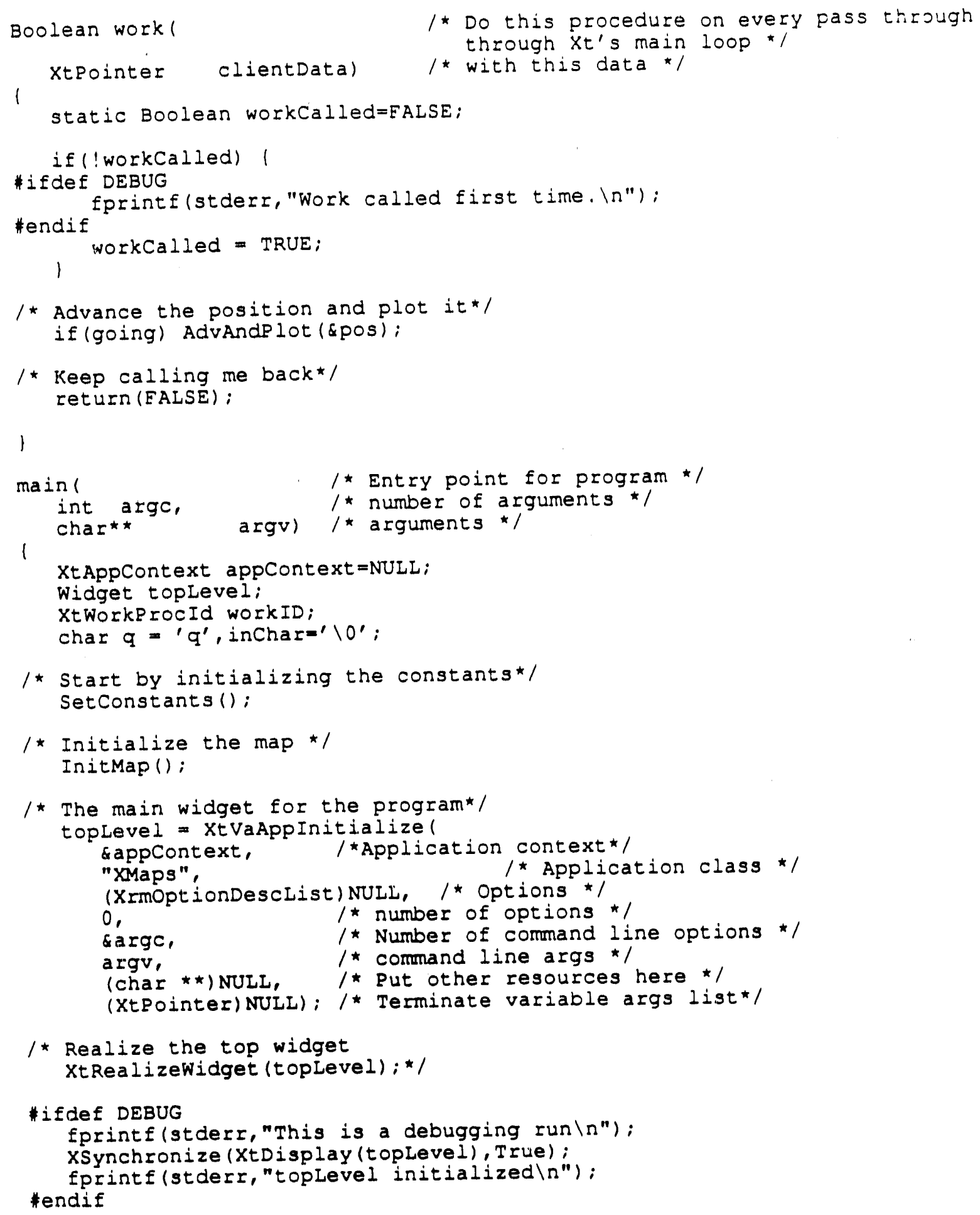


"sosw", /*The name of the plot*/

toplevel, /* The parent of this window */

ReceiveClick); /*Routine to receive click*/

*ifdef DEBUG
Eprinte(stderr," Surface of section initialized and realized $\backslash n "$ ": *endif

/* Register the error procedure

XtAppSetErrorHandler (appContext, MyError); ${ }^{*}$

/* Register the work precedure*/

workID = XtAppAddWorkProc (appContext, work, (XtPointer) NULL);

/ Realize the top widget */

XtRealizeWidget (toplevel);

1* Loop for events. */

XtAppMainLoop (appContext); 


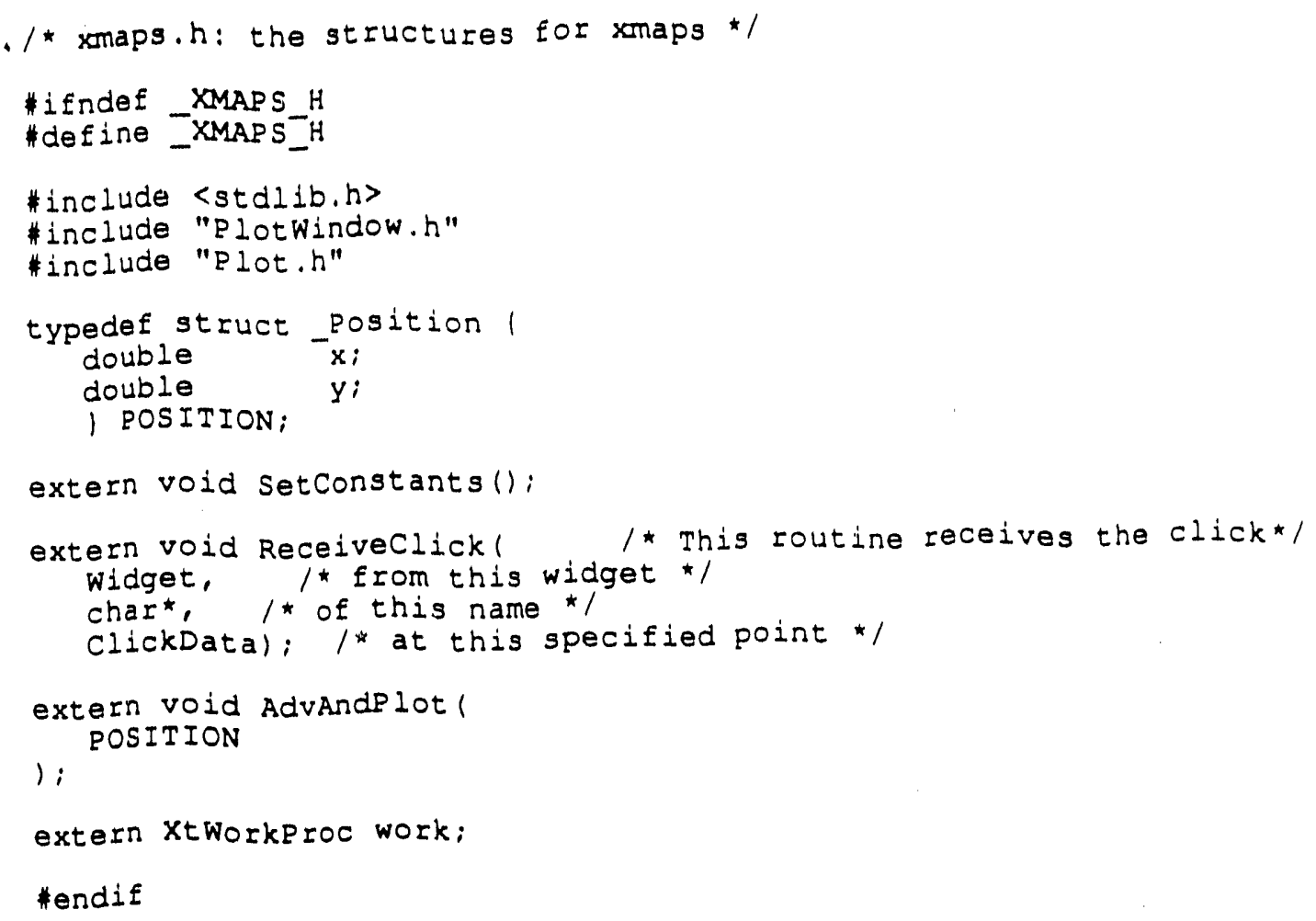




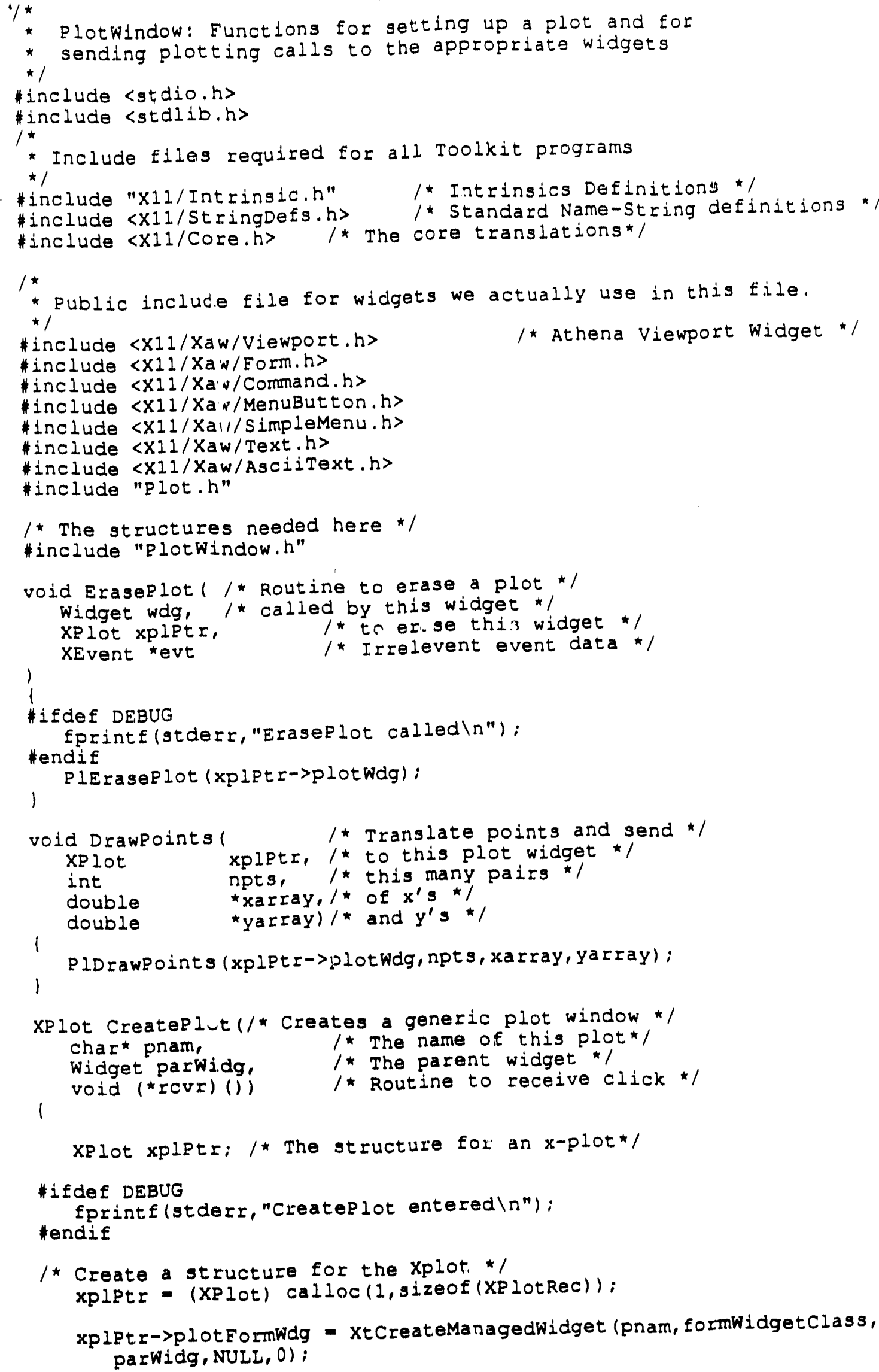


. \#ifdef DEBUG

fprintf(stder, "CreatePlot: plotForm widget created $\backslash \Omega$ ");

*endif

1* Create a command widget inslde this form */

xplPtr $>$ cmdWdg = XtVaCreateManagedWidget ("cmdWdg", commandwidgetClass,

xplptr->plotFormWdg, / This is a child of plotEormWdg*

xtNlabel, "Erase", $/$ * of this name */

XtNfromiloriz, NULL, /* Spaced to left side */

XtNhorizDistance, 5, /* By 10 pixels */

xtNleft, XtChainleft, / Keep this distance constant */

XtNright, XtChainleft, /* Keep this distance constant */

XtNfromvert, NULL, $/$ * Spaced from top*

XtNvertDistance, 5, /* By 10 pixels */

XtNtop, XtChainTop, / Keep this distance constant * /

xtNbottom, xtChainTop, / Keep this distance constant */

Hifdef

fprintf(stderr,"Createplot: cmdWdg created $\backslash n "$ ):

*endif

/* Create a viewport inside this form */

xplptr->vportWdg = XtVaCreateManaijedWidget ("vportWdg", viewportWidgetClass,

xplptr $\rightarrow$ plotEormWdg, / This is a child of plotFormWdg*/

XtNfromHoriz, NULI, /* Spaced to left side */

xtNhorizDistance, 5, /* By * pixels */

xtNleft, xtChainleft, /* Keep this distance constant */

XtNright, XtChainRight, /* Keep this distance constant */

XtNfromvert, xplptr->cmawdg, /* Spaced from command widget *

XtNvertDistance, 5, /* By * pixels */

XtNtop, XtChainTop, /* Keep this distance constant */

xtNbottom, xtChainBottom, /* Keep this distance constant */

xtNallowhoriz, True, $/$ * Allow horizontal scrollbars */

XtNallowvert, True, $\quad$ * Allow vertical scrollbars *

xtNuseRight, True, /* Put vertical scroll bar on the right */

xtNuseBottom, True, / Put horizontal scroll bar on the right */

NULI):

\#ifdef DEBUG

fprintf(stderr, "CreatePlot: vportWdg created $\backslash n "$ ); *endif

1* Create a plot inside this viewport */

xplPtr->plotwdg = (PlotWidget) XtVaCreatemanagedWidget ("plotwdg",

plotwidgetClass, xplPtr->vportWdg,

NULL):

* ifdef DEBUG

*endif

* * Put in the handler for the clicks */

xtAddCallback

(Widget) (xplptr->plotwdg),

PlNclickHandler,

( $x$ tCallbackProc) rcvr,

(XtPointer) NULL) ;

* Add a callback *

/* to this widget */

* to handle clicks * 1

/* by calling this function */

/* passing this data. */

*ifdef DEBUG

*endif

forintf(stderr, "Createplot: finished initialization of $8>(n ", p n a m)$;

/ Now add a caliback to the Erase button to send to the plotwidget */

$x t$ AddCallback ( 1 * Add a callback */

(Widget) (xplptr->cmdWdg), /* to this widget * 1

XtNcallback,

/ * to handle clicks */

(XtCallbackProc) ErasePlot,

(Xtpointer) xplptr):

$/$ * by calling this function */

/* passing this data. */

* Realize these widgets 
XtRealizeWidget (xplptr $\rightarrow$ plotEormWdg) ; $/$ lifdef DEBUG \#endif

/ * Return the value of the created widget* / 1 return (xpletr); 


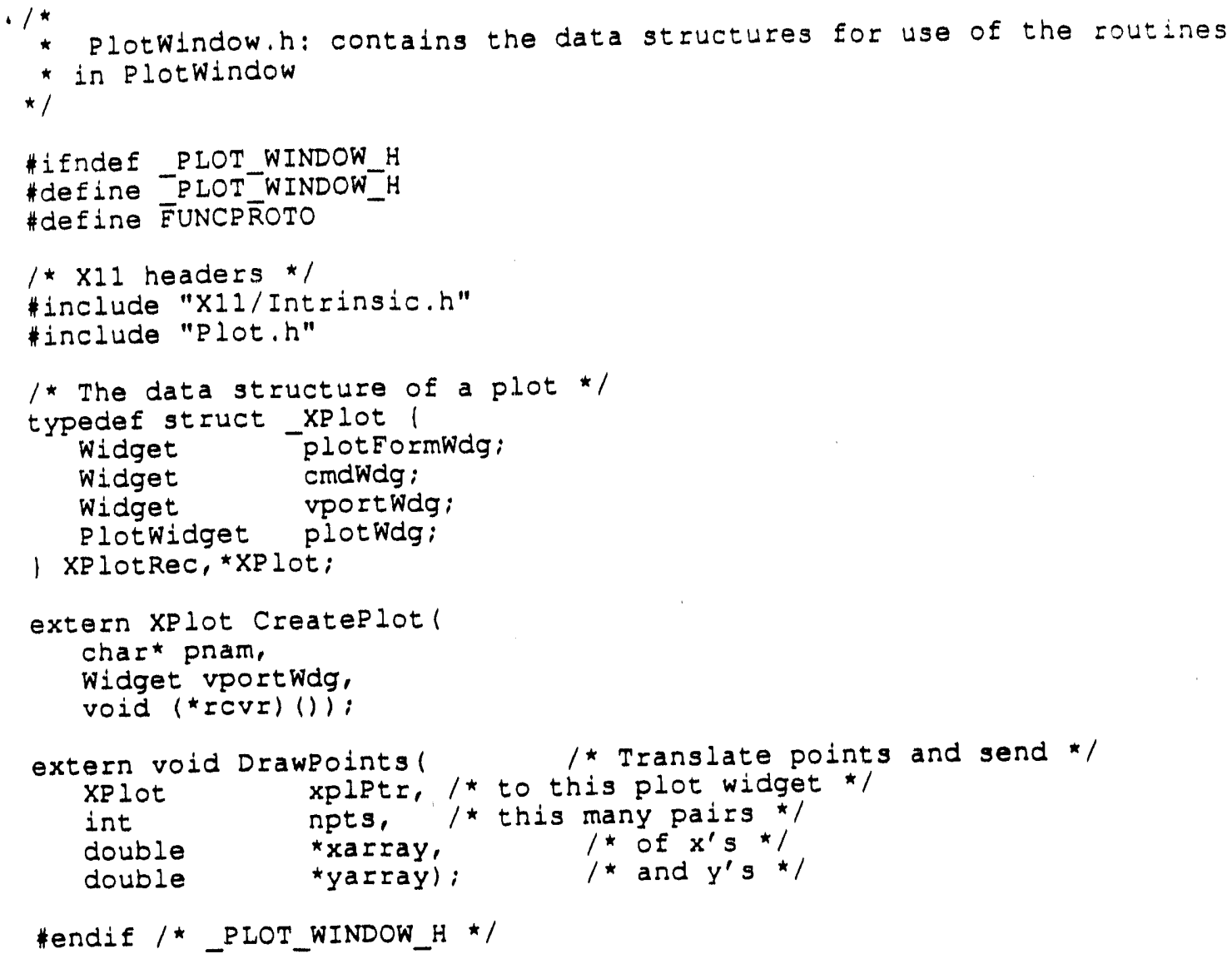




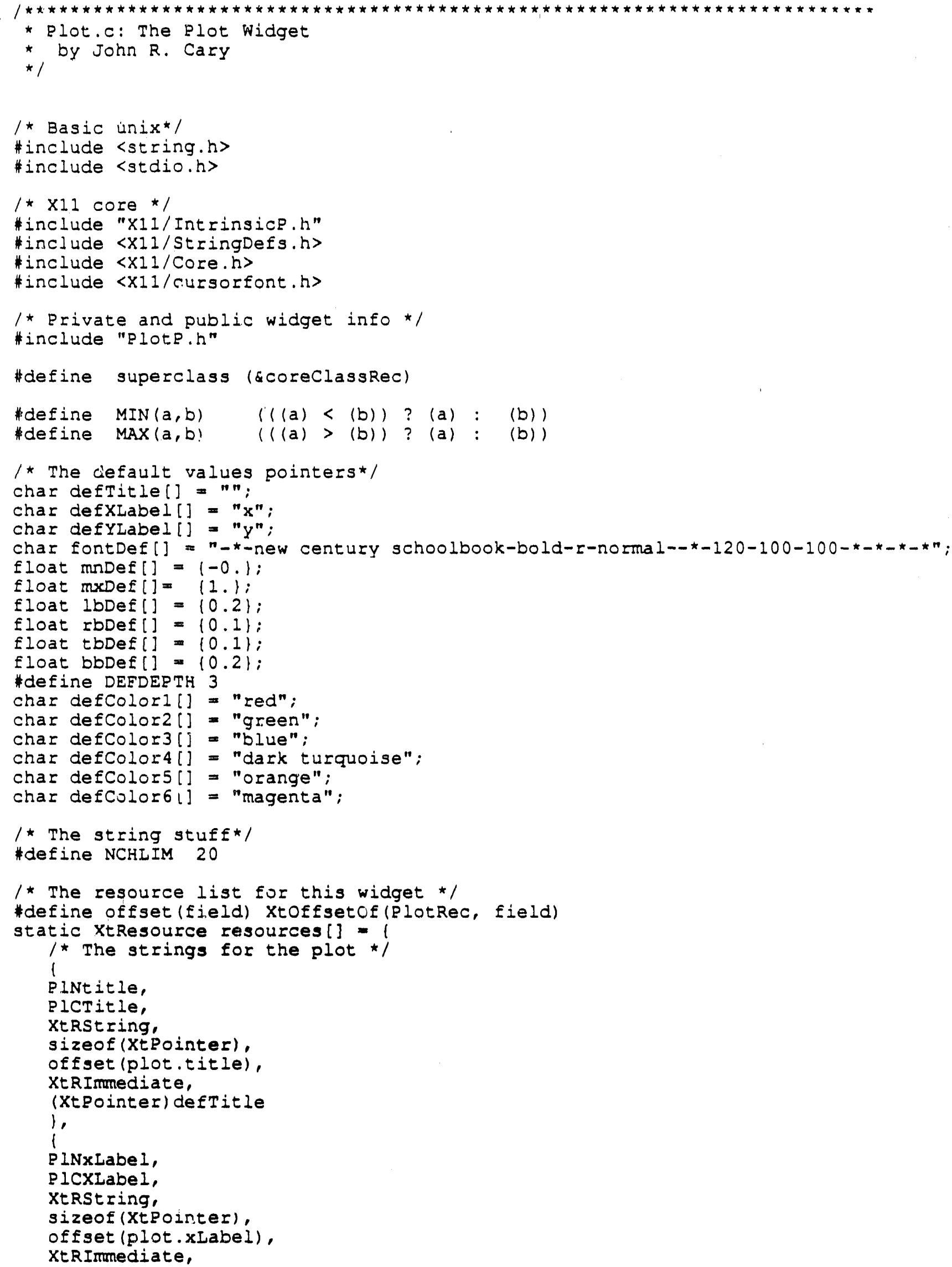




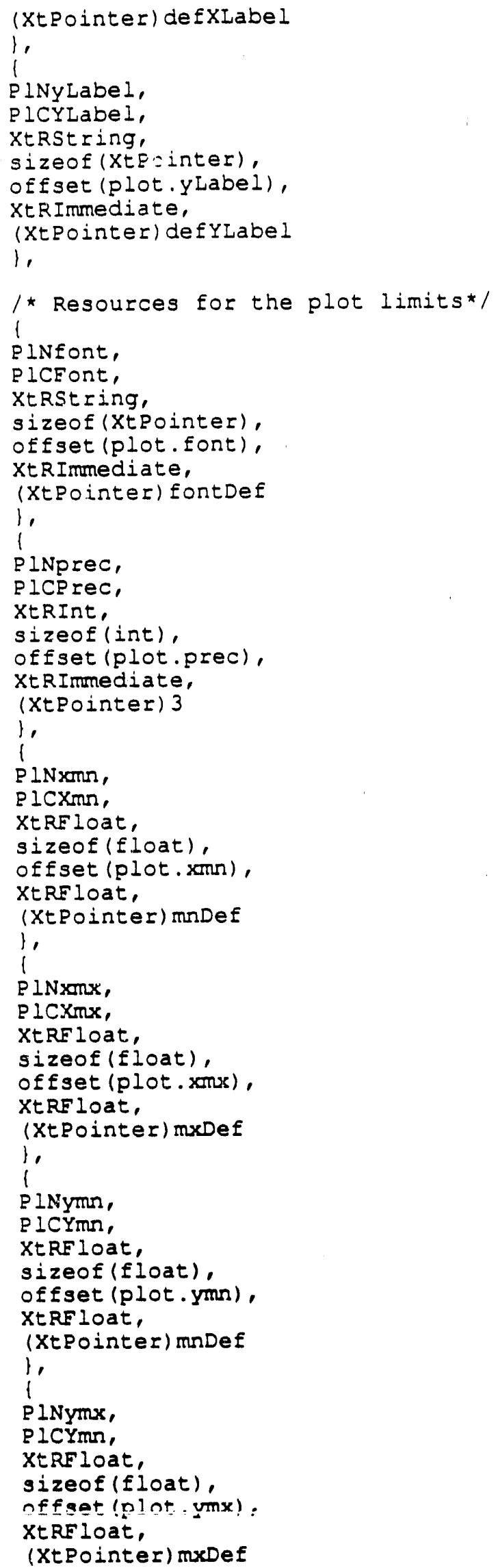




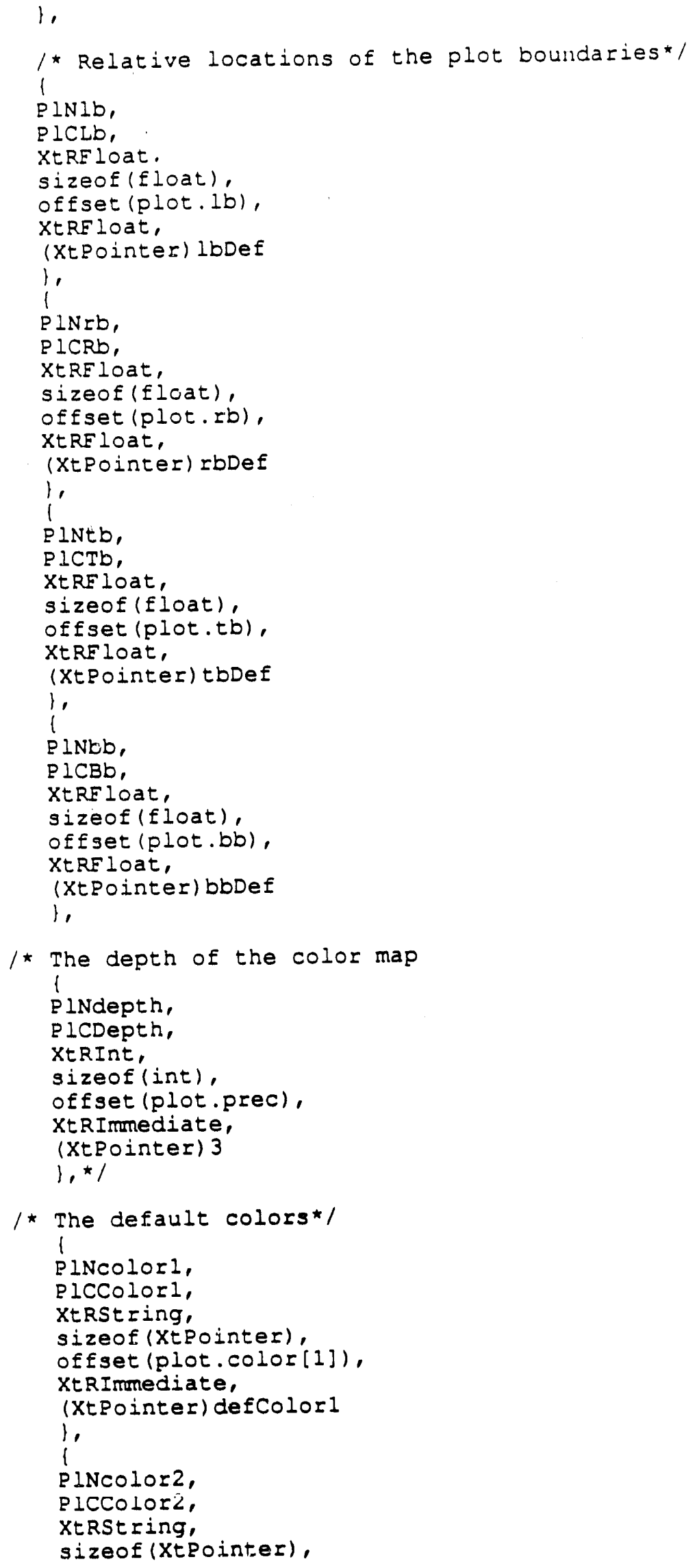




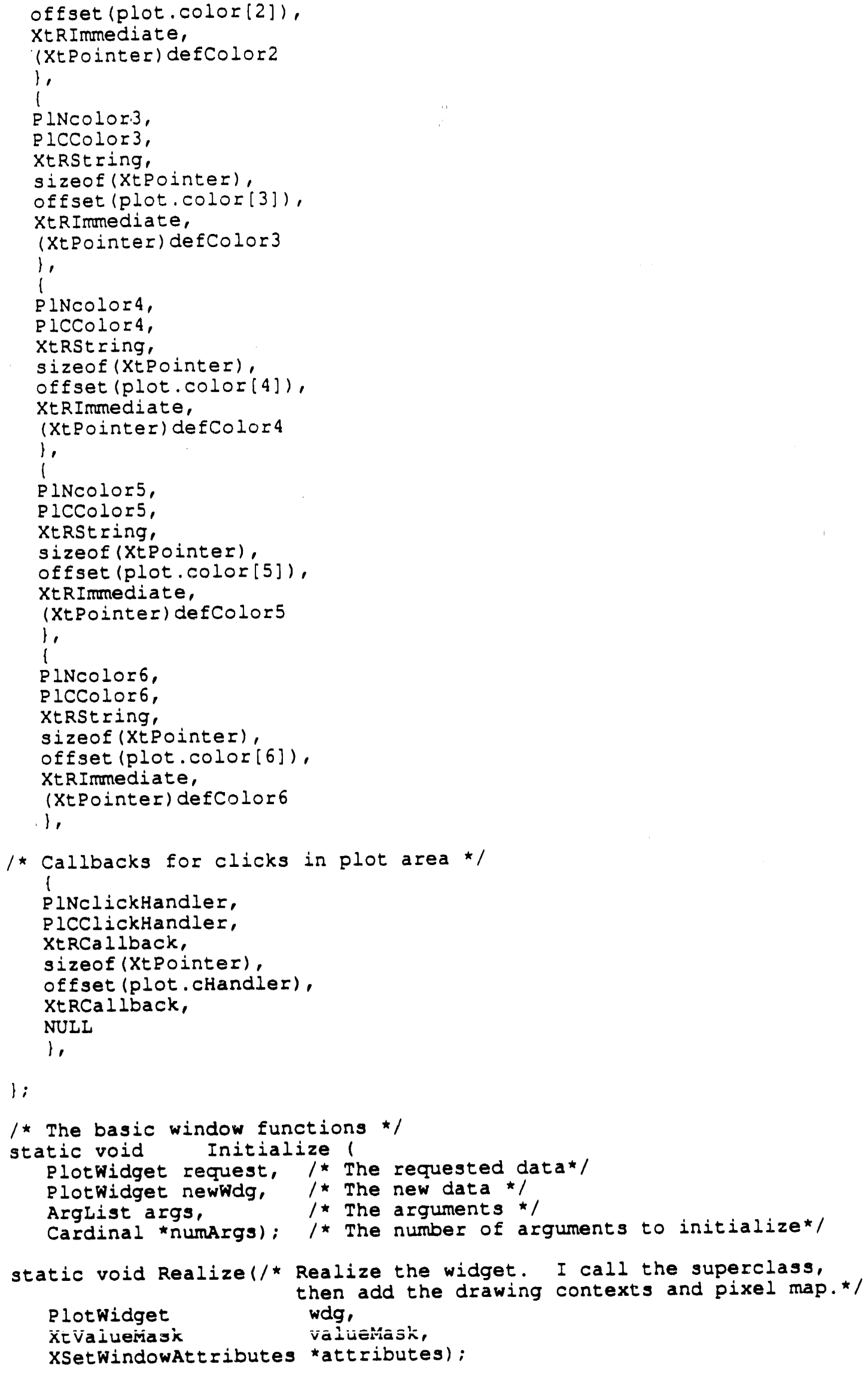




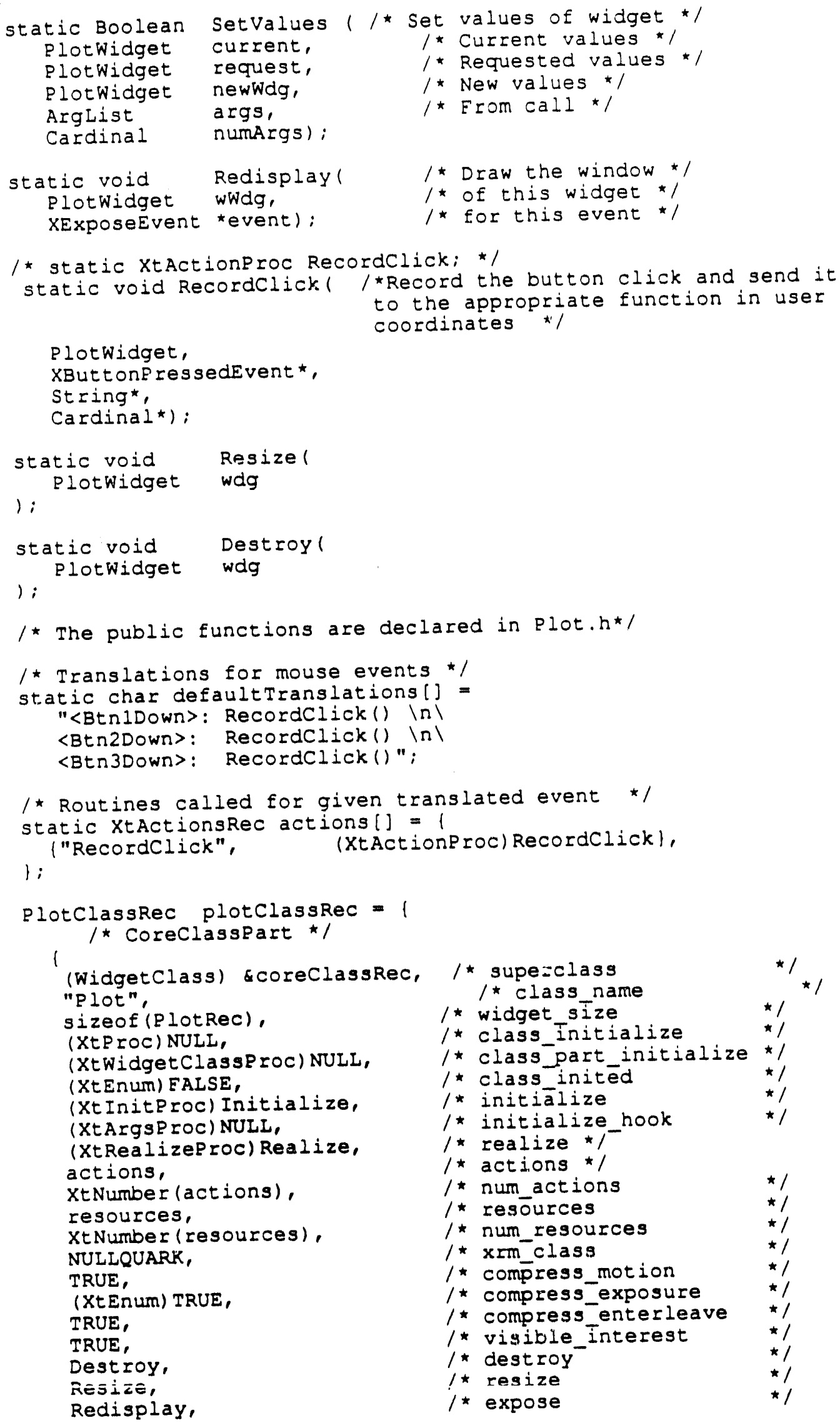

Plotwidget,

XBut onf ressedEvent *,

String*,

Cardinal*);

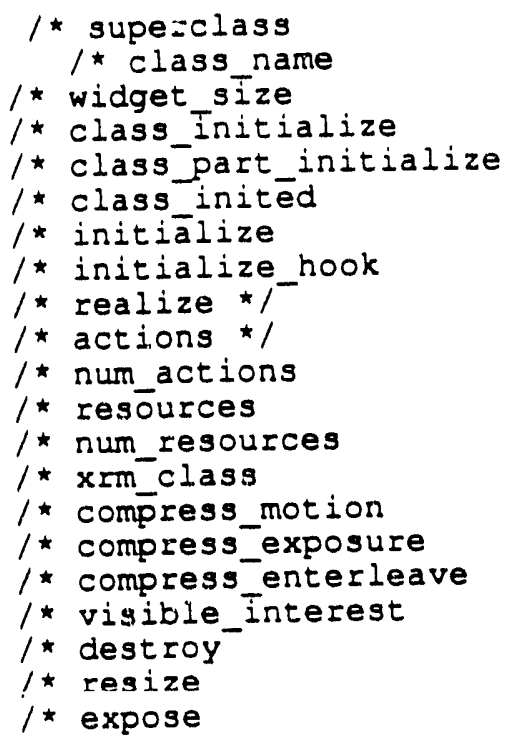




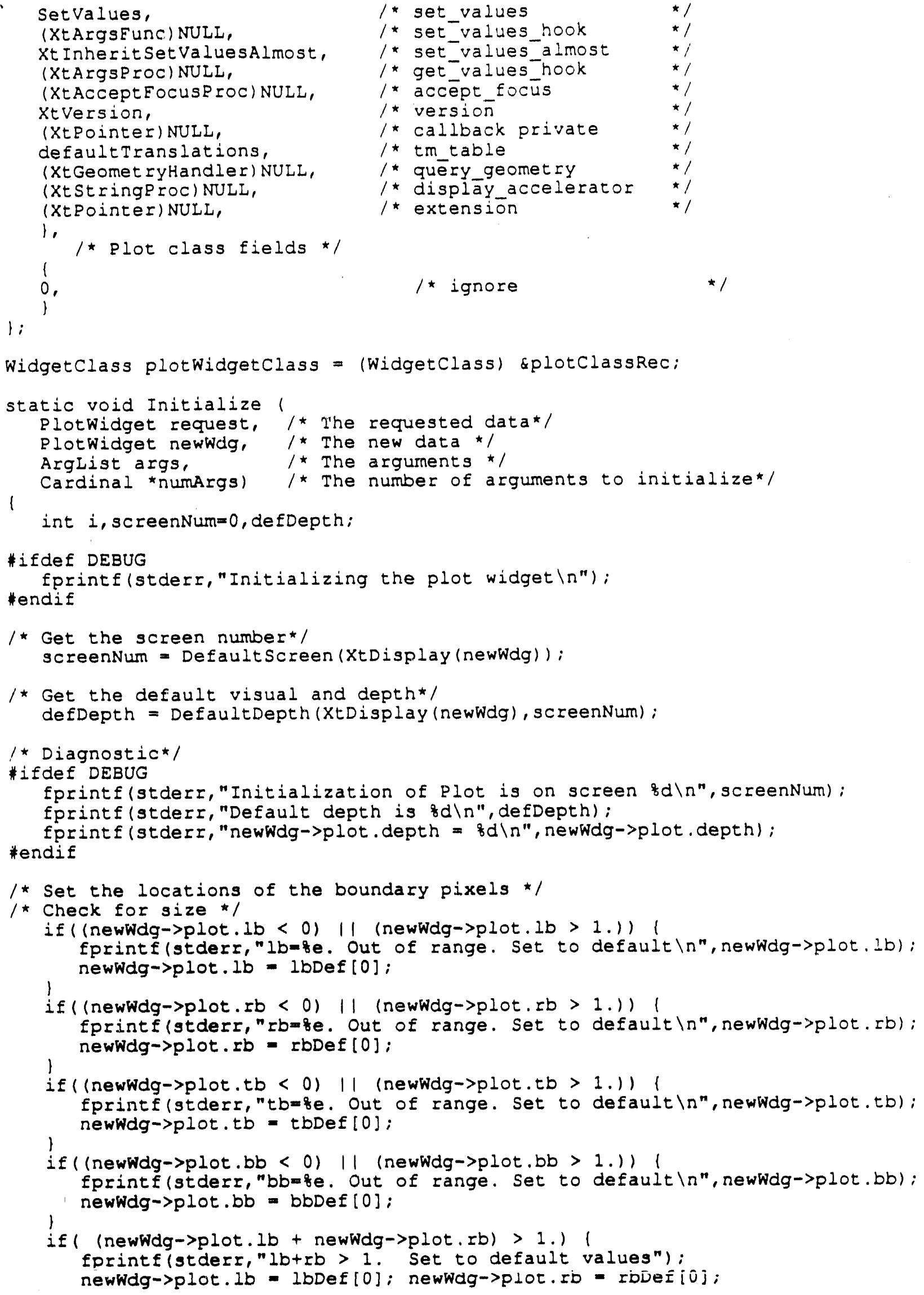




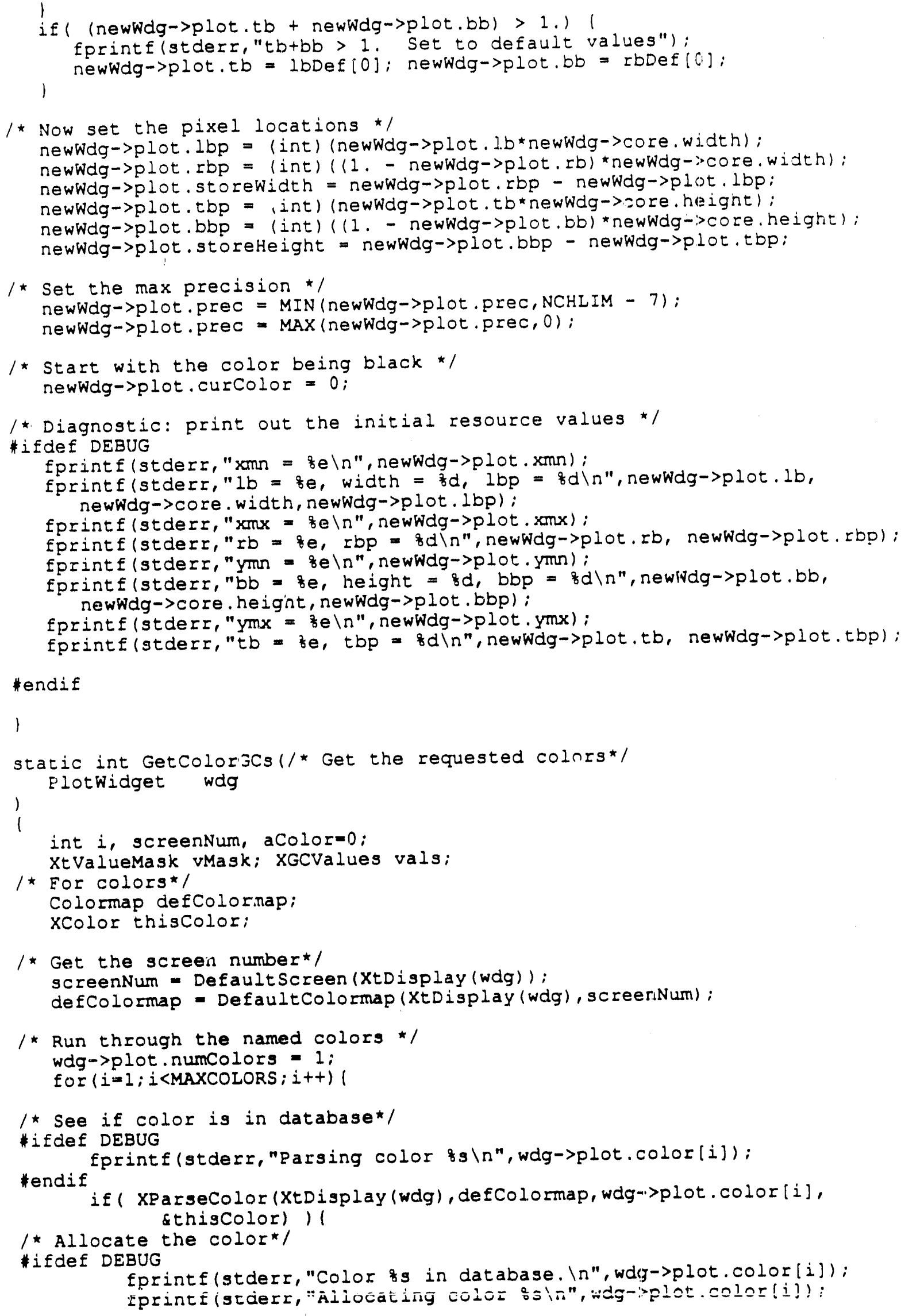


*endif

if( XAllocColor (XtDisplay (wdg), defColormap, athisColor) /1

\#ifdef DEBUG

\#endif

fprintf(stderr,"Color zo allocated. In",wdg->plot.color(ij):

\#ifdef DEBUG

vals.foreground = thiscolor.pixel:

vals.background = WhitePixel (XtDisplay (wdg), screenNum):

vals.font $=$ wdg->plot. fontID:

vMask = GCEoreground | GCBackground | GCFont;

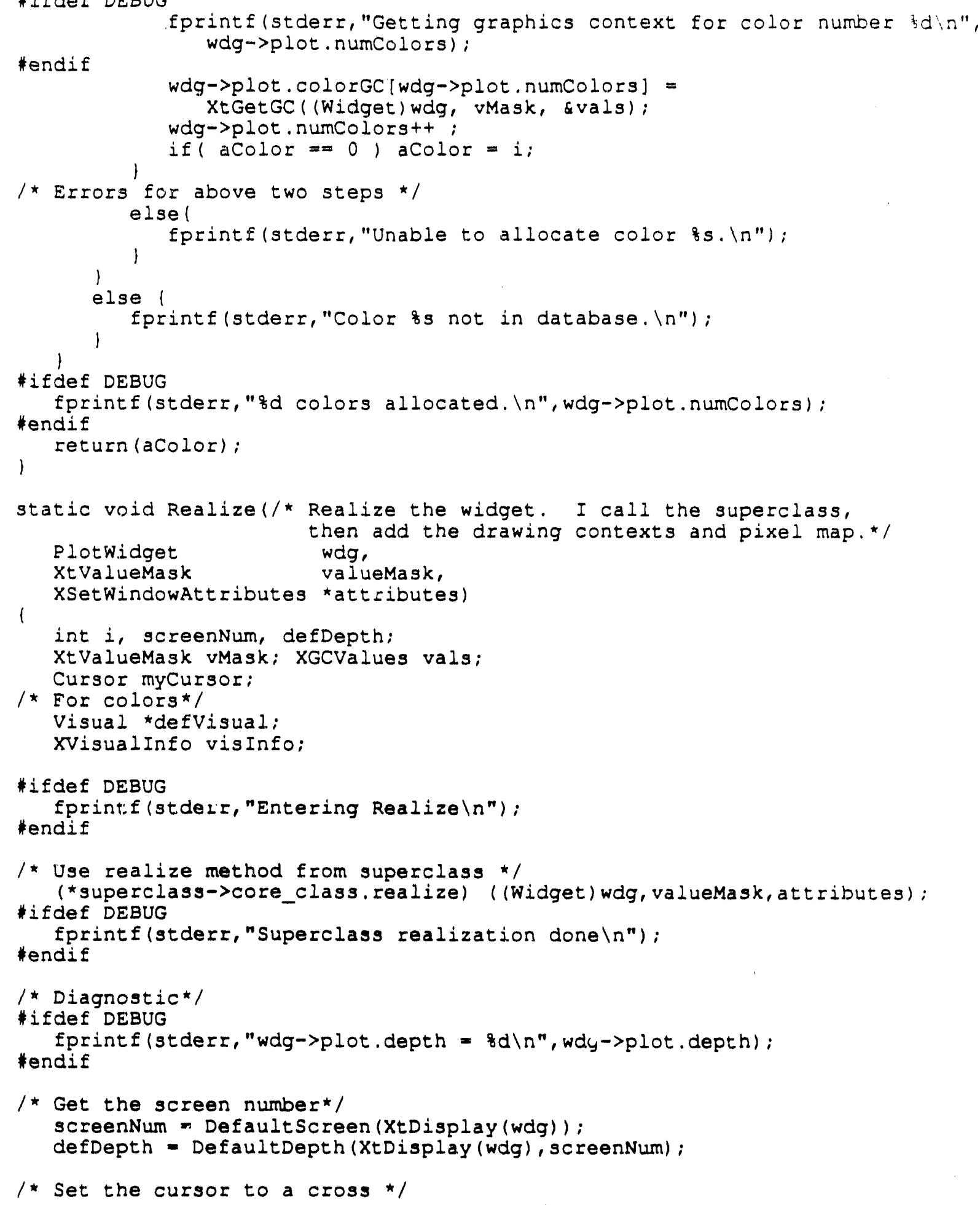


/*myCursor = XCreateEontCursor(XtDisplay (wdg), XC_crosshair); * myCursor = XCreateE ontCursor(XtDisplay (wdg), XC_tCross):

xDefineCursor ( $x t D i s p l a y(w d g), x t w i n d o w(w d g)$, myCursor);

1* Get the font for the drawing context*/ wdg->plot', fontID = XLoadFont (XtDisplay (wdg), wdg->plot, font);

*ifdef DEBUG fprintf(stderr, "Font loaded $\backslash n$ ");

*endif

if ( wdg->plot. font ID $=0$ )

Eprintf(stderr, "Unable to load font कs $\backslash n$ ", wdg->plot.font):

1* We use xtGetgC for the erasegC (white) because it is fixed */ vals.foreground = WhitePixel (XtDisplay (wdg), screenNum); vals.background = BlackPixel (XtDisplay (wdg), screenNum); vMask = GCForeground | GCBackground ; wdg->plot. eraseGC = xtGetGC ( (Widget) wdg, vMask, \&vals):

* ifdef DEBUG fprintf (stderr, "Erase context made $\backslash n "$ ")

\#endif

/* The black graphics context is the first color context * / vals.foreground = BlackPixel (XtDisplay (wdg), screenNum); vals.background = WhitePixel (XtDisplay (wdg), screenNum); vals. font $=$ wdg->plot. font ID:

vMask = GCForeground | GCBackground | GCFont; wdg->plot. colorGC $[0]=$ xtGetGC ((Widget) wdg, vMask, \&vals):

* ifdef DEBUG *endif fprinte (stderr, "Black context made $\backslash n$ "):

1 * Get the other colors */ if (defDepth>1) 1

$i=5 ;$

while( XMatchVisualinfo (XtDisplay (wdg), screenNum, defDepth, /*visual class*/ i--, Gvisinfol):

*ifdef DEBUG

*endif fprinte(stderr,"Visual of number of found $\backslash n ",++i$ ):

if( $i$ <taticColor) 1 eprinte(stderr,"No color visual found. Will use black and white. $\ln "$ ): \} goto EndColor:

\#ifdef DEBUG

*endif eprintf(stderr,"Allocating colors $\backslash n "$ ");

1 wdg $\rightarrow$ plot. curColor = GetColorgCg (wdg) - 1;

else wdg->plot. numColors = 1;

EndColor:

/ Create and erase the pixel map for the drawing*/

*ifdef DEBUG

fprinte (stderr, "Creating id $x$ id pixmap", wdg->plot. storewidth, wig $>$ plot . storeHeight): fprinte (stderr," of depth od $\backslash n^{n}$, defDepth);

*endif

wdg->plot.pixmap = XCreatePixmap (

XtDisplay (wdg), / the display for this pixmap */

xtwindow(wdg), /*The window of this pixmap */

wdg->plot.storewidth, /*the width of the pixmap */

wdg->plot.storeHeight, / *the height of the pixmap */

- ifdef DEBUG 
- Eprintf(stderr, "Plxmap created $\backslash n$ ");

*endif

/* Erase the pixmap */

XEillRectangle (

XtDisplay (wdg),

wdg $\rightarrow$ plot.pjixmap,

wdg $\rightarrow$ plot . eraseGC,

$0,0,1$ * Erom upper left corner *

wdg->plot.storewidth, /* the width of the pixmap */

wdg->plot. storeHeight); $/$ the helght of the pixmap *

1

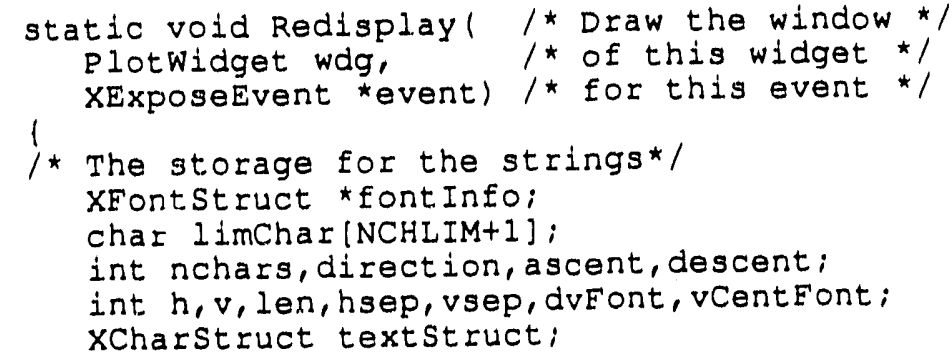

*ifdef DEBUG

fprintf (stderr, "Entering Redisplay $\backslash n "$ ):

\#endif

1* Make sure the widget exists

Lf (!XtIsRealized(wdg)) return; */

/ D Draw a rectangle around the plotting region*/

/* Note that one must diminish the location by 1 and increase

the width and height by one to prevent overwriting * $/$

\#ifdef DEBUG

fprintf(stderr, "About to draw the rectangle $\backslash n$ "):

\#endif

XDrawRectangle (XtDisplay (wdg), XtWindow (wdg),

wdg $\rightarrow$ plot. colorGC[0],

wdg $\rightarrow$ plot. Ibp - 1, wdg->plot, tbp - 1,

wdg->plot. storewidth +1 , wdg->plot. storeheight +1 );

1* Draw the limits of the plot */

wdg $\rightarrow$ plot.prec $=$ NCHIIM $-6 ; /$ the displayed precision $/$

fontInfo = XQueryFont (XtDisplay (wdg), wdg->plot. font ID);

dvFont = fontInfo->ascent + fontInfo->descent:

vCentFont = (fontInfo->ascent - fontInfo->descent) /2;

vsep = fontInfo->ascent $/ 2$ + fontInfo->descent:

hsep = fontInfo->ascent $/ 2$ + fontInfo->descent;

/* Each limit * /

sprintf (limchar,"o. 3g",wdg->plot.xmn);

len $=$ strlen (limChar) $;$

$h=$ wdg->plot.1bp - XTextWidth (Eunt Info, limChar, len) /2;

$v=$ wdg->plot.bbp + vsep + dvFont;

XDrawstring (XtDisplay (wdg), Xtwindow (wdg), wdg->plot. colorGC[0],

$h, v, l i m C h a r$, len):

sprintf (limChar, "q. $3 \mathrm{~g}^{\prime \prime}, \mathrm{wdg}->$ plot . xmx) ;

len = strlen (limChar);

$\mathrm{h}=$ wdg->plot.rbp - X'Textwidth (fontInfo, limChar, len) /2;

XDrawString (XtDisplay (wdg), Xtwindow (wdg), wdg->plot. colorGC $[0]$,

$h, v$, limChar, len);

sprintf (limChar,"o. 3g",wdg->plot.ymur):

len = strlen (limChar);

$h=w d g->p l o t .1 b p-x T e x t W i d t h$ (fontInfo, limChar, len) - hsep;

$v=$ wdg->plot.bbp + (fontinfo->ascent - fontInfo->descent) $/ 2$; 


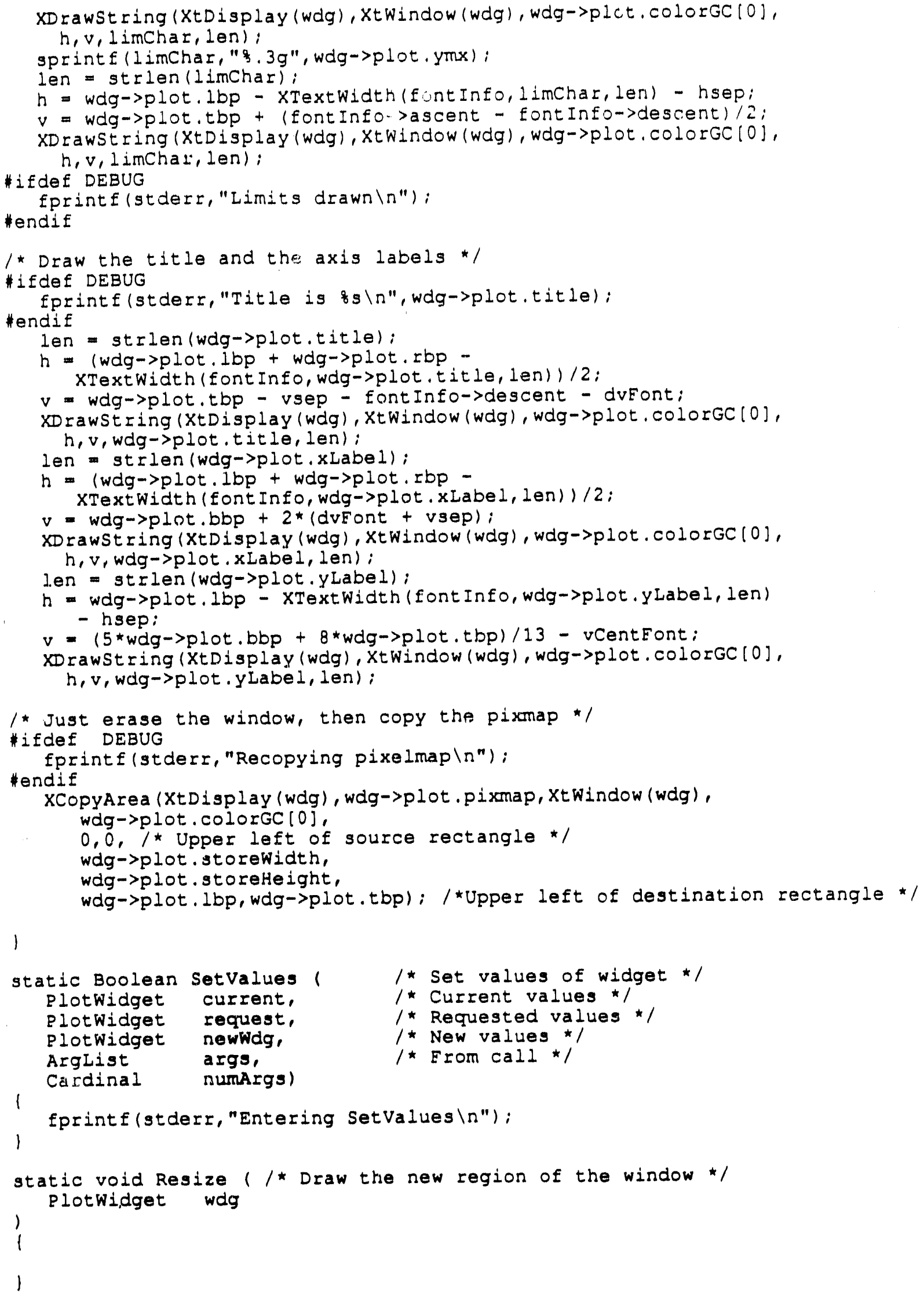




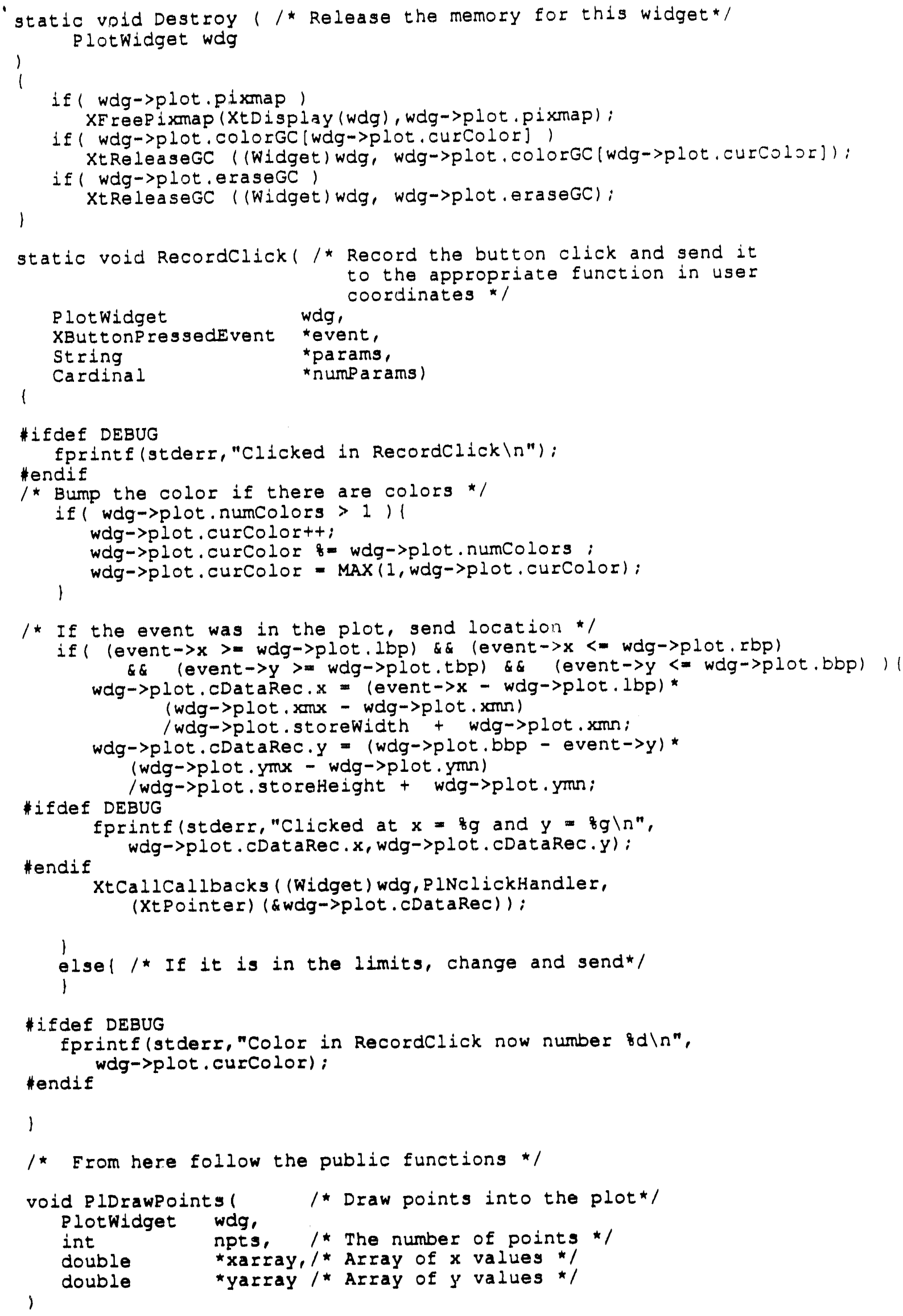




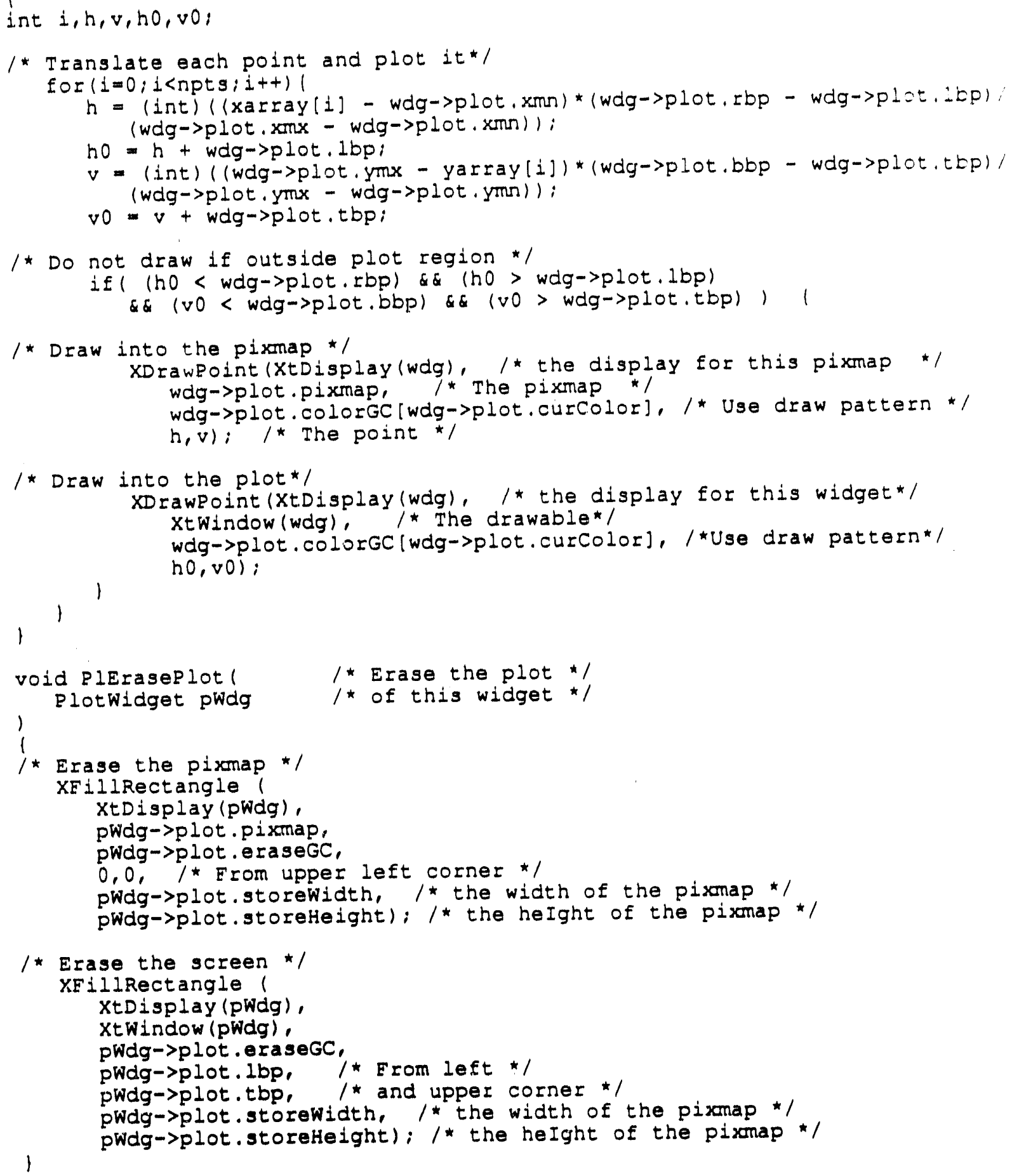




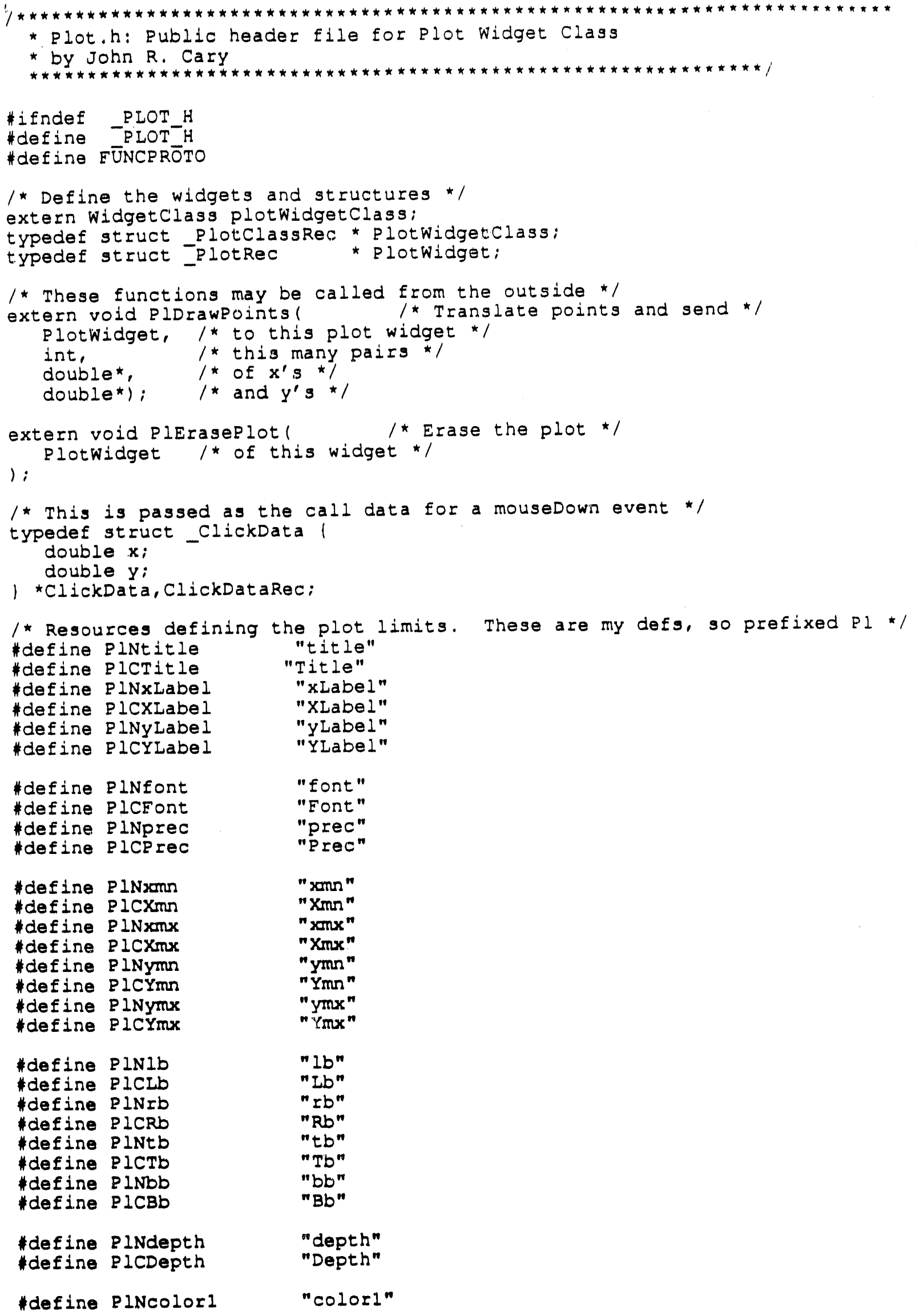




\begin{tabular}{|c|c|c|}
\hline $\begin{array}{l}\text { \#define } \\
\text { \#define } \\
\text { \#define } \\
\text { \#define } \\
\text { \#define } \\
\text { \#define } \\
\text { \#define } \\
\text { \#define } \\
\text { \#define } \\
\text { \#define } \\
\text { \#define }\end{array}$ & 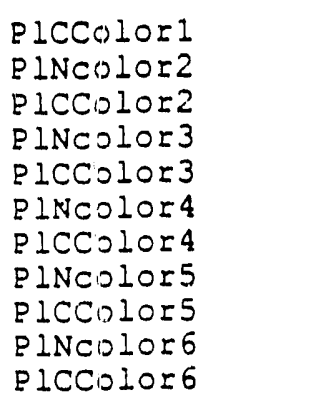 & $\begin{array}{l}\text { "colorl" } \\
\text { "color2" } \\
\text { "Color2" } \\
\text { "color3" } \\
\text { "Color3" } \\
\text { "color4" } \\
\text { "color4" } \\
\text { "color5" } \\
\text { "Color5" } \\
\text { "color6" } \\
\text { "color6" }\end{array}$ \\
\hline te & $\begin{array}{l}\text { INCLickHandler } \\
\text { ICCLickHandler }\end{array}$ & $\begin{array}{l}\text { ClickHandler" } \\
\text { ClickHandler" }\end{array}$ \\
\hline
\end{tabular}

*endif $/ *$. DRAWRGN_H */ 


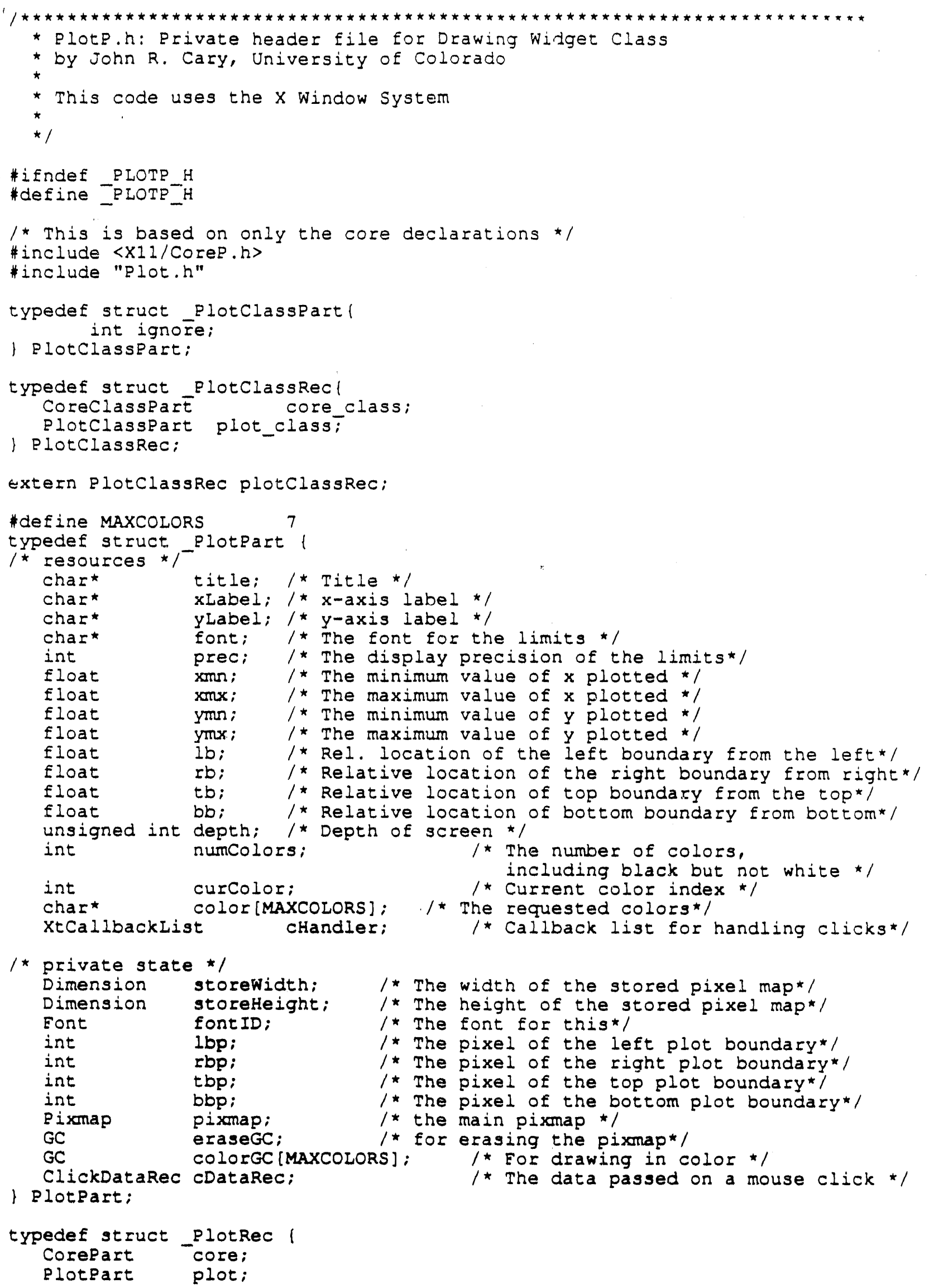


i) PlotRec:

*endif /* _PLOTP_H */ 

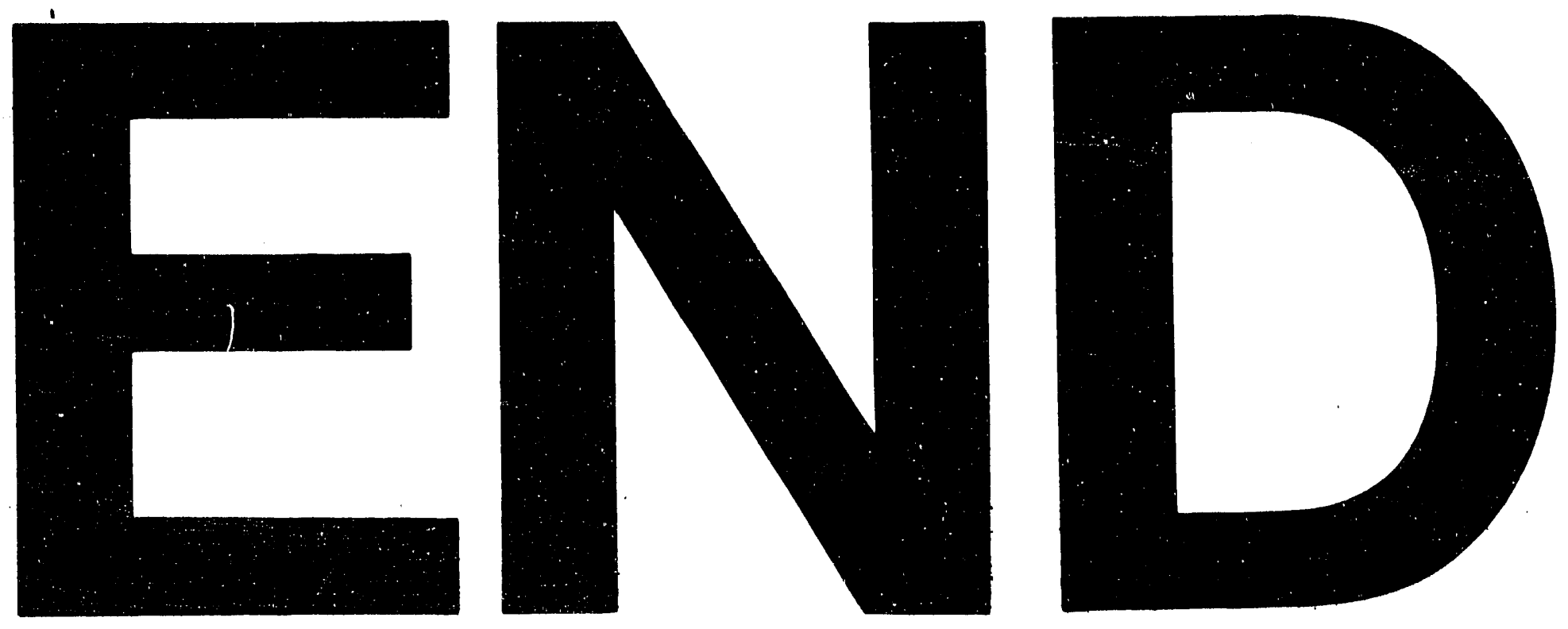

“
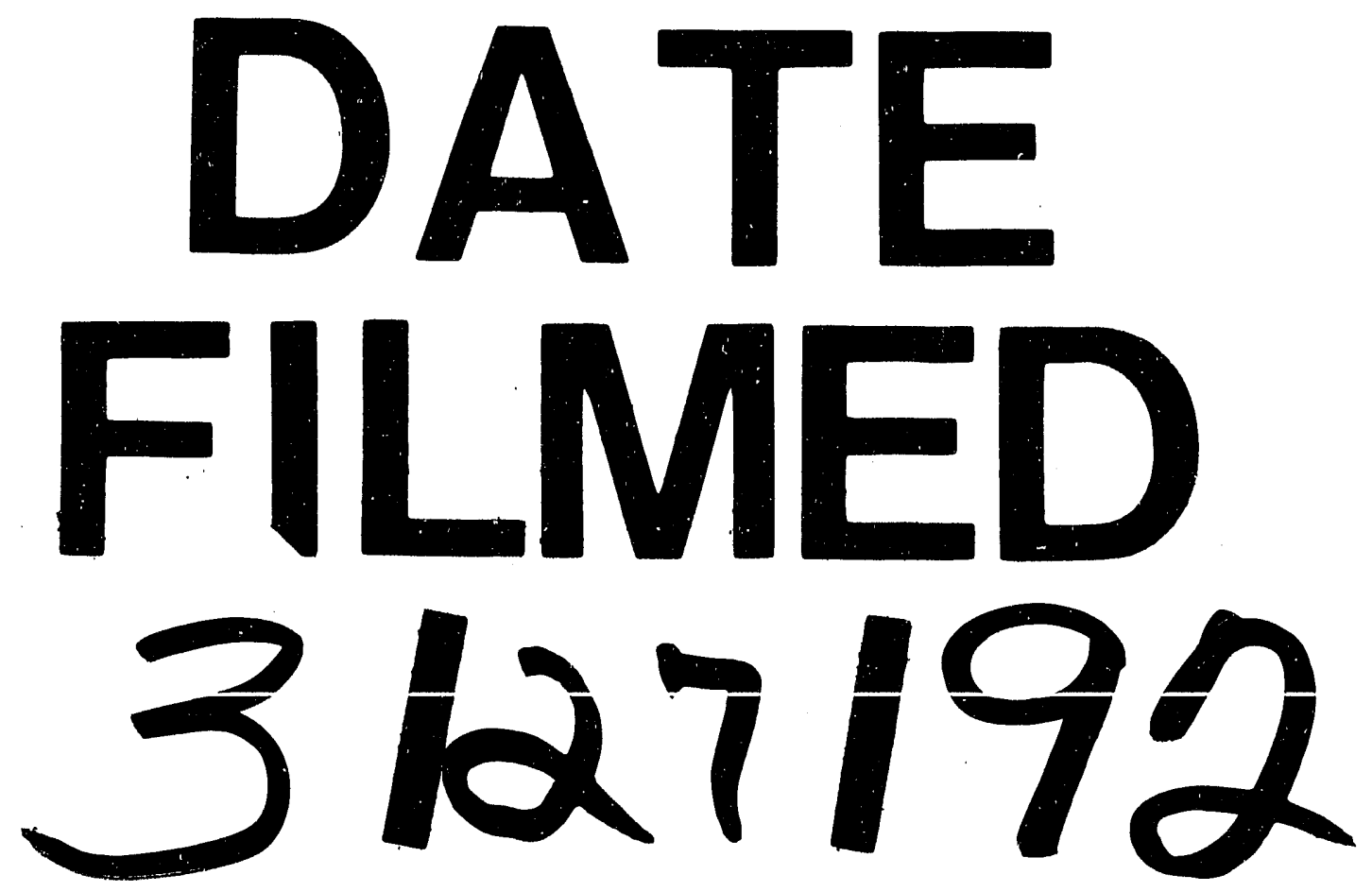

$I$ 


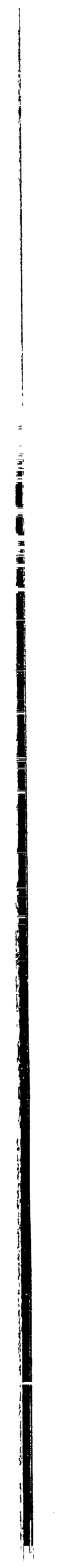

